\title{
Evolution of oceanic near surface stratification in response to an autumn storm
}

Article

Accepted Version

Lucas, N. S., Grant, A. L. M., Rippeth, T. P., Polton, J. A., Palmer, M. R., Brannigan, L. and Belcher, S. E. (2019) Evolution of oceanic near surface stratification in response to an autumn storm. Journal of Physical Oceanography, 49 (11). pp. 2961-2978. ISSN 1520-0485 doi:

https://doi.org/10.1175/jpo-d-19-0007.1 Available at https://centaur.reading.ac.uk/86803/

It is advisable to refer to the publisher's version if you intend to cite from the work. See Guidance on citing.

To link to this article DOI: http://dx.doi.org/10.1175/jpo-d-19-0007.1

Publisher: American Meteorological Society

All outputs in CentAUR are protected by Intellectual Property Rights law, including copyright law. Copyright and IPR is retained by the creators or other copyright holders. Terms and conditions for use of this material are defined in the End User Agreement. 


\section{CentAUR}

Central Archive at the University of Reading

Reading's research outputs online 


\title{
Evolution of Oceanic Near Surface Stratification in Response to
}

\author{
an Autumn Storm \\ Natasha Sarah Lucas* ${ }^{\dagger}$ \\ School of Ocean Sciences, Bangor University, Gwynedd, UK \\ Alan Laurence Michael Grant ${ }^{\dagger}$ \\ University of Reading, Reading, $U K$ \\ Tom Rippeth \\ School of Ocean Sciences, Bangor University, Bangor, Gwynedd, UK
}

Jeff A. Polton, Matthew Palmer

National Oceanography Centre, Liverpool, UK

Liam Brannigan

MISU, Stockholm University

Stephen E. Belcher

Met Office, Exeter, UK

${ }_{15}^{*}$ Corresponding author address: School of Ocean Sciences, Bangor University, Gwynedd, UK

16 E-mail: n.lucas@bangor.ac.uk

Generated using v4.3.2 of the AMS LATEX template

1

Early Online Release: This preliminary version has been accepted for publication in Journal of the Physical Oceanography, may be fully cited, and has been assigned DOI 10.1175/JPO-D-19-0007.1. The final typeset copyedited article will replace the EOR at the above DOI when it is published.

(C) 2019 American Meteorological Society 
${ }_{17}^{\dagger}$ These authors contributed equally to this work 


\begin{abstract}
${ }_{23}$ Mixing, Ocean Submesoscale Interaction Study (OSMOSIS), are presented.

${ }_{24}$ The focus of this study is a storm which passed over the observational area

Understanding the processes that control the evolution of the ocean surface boundary layer (OSBL) is a prerequisite for obtaining accurate simulations of air-sea fluxes of heat and trace gases. Observations of the rate of dissipation of turbulent kinetic energy $(\varepsilon)$, temperature, salinity, current structure and wavefield over a period of 9.5 days in the NE Atlantic during the Ocean Surface during this period. The profiles of $\varepsilon$ in the OSBL are consistent with profiles from large eddy simulation (LES) of Langmuir turbulence. In the transition layer (TL), at the base of the OSBL, $\varepsilon$ was found to vary periodically at the local inertial frequency. A simple bulk model of the OSBL and a parametrisation of shear driven turbulence in the TL are developed. The parametrisation of $\varepsilon$ is based on assumptions about the momentum balance of the OSBL and shear across the TL. The predicted rate of deepening, heat budget and the inertial currents in the OSBL were in good agreement with the observations, as is the agreement between the observed value of $\varepsilon$ and that predicted using the parametrisation. A previous study reported spikes of elevated dissipation related to enhanced wind-shear alignment at the base of the OSBL after this storm. The spikes in dissipation are not predicted by this new parametrisation, implying that they are not an important source of dissipation during the storm.
\end{abstract}




\section{Introduction}

The ocean surface boundary layer (OSBL) is a critical interface in the Earth system, through which heat, freshwater, momentum and trace gases are fluxed between the atmosphere and the ocean (Belcher et al. 2012; Rippeth et al. 2014). Because of its importance, there is strong interest in understanding the processes that determine the characteristics and evolution of the OSBL (e.g. Kilbourne and Girton (2015); Aijaz et al. (2017)).

Mixing by turbulence in the OSBL tends to produce a layer with relatively weak vertical gradients in temperature and salinity which will be referred to as the well-mixed layer (WML). The WML is separated from the deeper ocean by a stratified transition layer (TL). Current shear across the transition layer may be associated with near inertial waves (NIW) (Plueddemann and Weller 1999), which are a ubiquitous feature of the surface ocean (Pollard 1980; D'Asaro 1985), and are a significant energy source driving turbulent mixing in the ocean (Alford 2003; Watanabe and Hibiya 2002). The generation of inertial motions in the OSBL is highly intermittent, with storms providing an important source of energy(D'Asaro 1985; Large and Crawford 1995).

The shear associated with the NIW is concentrated across the stratified transition layer (Pollard and Millard 1970; D'Asaro 1985), which is often in a state of marginal stability, with a Richardson number $\mathrm{O}(1)$, so that the shear may result in the generation of turbulence in the TL (Johnston and Rudnick 2009; Rippeth et al. 2005, 2009). Observations reported by Burchard and Rippeth (2009); Lenn et al. (2011); Brannigan et al. (2013); Lincoln et al. (2016) suggest that the generation of turbulence within the transition layer is a result of surface wind and current shear alignment which produces enhanced shear at the base of the WML. Using a one-dimensional model, Plueddemann and Farrar (2006) showed that the energy input into the NIW is balanced by the downward 
propagation of NIW energy, and the local dissipation due to shear generated turbulence within the TL.

Generation of turbulence by shear across the TL is particularly efficient when the rate at which near surface winds rotate due to the motion of a storm, matching the inertial period of the winddriven currents (Large and Crawford 1995; Dohan and Davis 2011; Chen et al. 2015). This resonance condition allows large shears to build at the base of the well-mixed layer, which can lead to the growth of a stratified shear layer below the WML, without signficant impact on the thickness of the WML (Dohan and Davis 2011; Johnston et al. 2016; Chen et al. 2016).

Skyllingstad et al. (2000) used large-eddy simulation (LES) to look at shear production in the TL for resonant and non-resonant situations. Compared to resonant conditions, the predicted current shear and turbulence were signficantly smaller for non-resonant conditions. Grant and Belcher (2011) have derived a parametrisation for the magnitude of the maximum shear production and dissipation at the base of the WML, due to resonant wind forcing.

Here we present measurements of the dissipation rate, $\varepsilon$, temperature and salinity of the water column, obtained by an Ocean Microstructure Glider over a period of 9.5 days. The measurements were obtained during the process cruise of the OSMOSIS (Ocean Surface Mixing and Submesoscale Interaction Study) project in the North East Atlantic in September 2012.

During the period of the observations a significant storm occurred. The aim of this study is to investigate the processes responsible for the evolution of the OSBL during the storm. To achieve this aim we will combine the profiles of $\varepsilon$ from the microstructure glider, with supporting data, to test a new parametrisation for OSBL mixing.

This paper is arranged as follows: Section 2 gives a description of the observational campaign together with the methods used to collect the data. Section 3 provides a description of the main 
experimental results, Section 4 describes the turbulence measurements in the transition layer, and Section 5 concludes the paper with a discussion of the key results.

\section{Observations and Modelling}

The observations used in this study were collected during a multidisciplinary cruise aboard the Royal Research Ship Discovery (Allen et al. 2012), as part of the NERC OSMOSIS project. The cruise took place in the vicinity of the Porcupine Abyssal Plain (PAP) observatory $\left(48.69^{\circ} \mathrm{N}\right.$, $16.19^{\circ} \mathrm{W}$ ) which is to the south west of the UK. The site is representative of the open ocean, with a water depth of $\sim 4800 \mathrm{~m}$. The measurements were made from the evening of the $17^{\text {th }}$ September 2012 , to the evening of the $27^{\text {th }}$ September (year days 260 to 270 ).

The observations to be discussed were obtained using: 1) an Ocean Microstructure glider (OMG); 2) a TRIAXYS directional wave buoy; 3) ship borne measurements of meteorological data and 4) water velocities from an ADCP.

\section{a. Ocean Microstructure Glider $(O M G)$}

The OMG was a Teledyne Webb Research Slocum coastal electric glider equipped with an unpumped SeaBird CTD sensor and a Rockland Scientific International MicroRider microstructure package. The OMG microstructure package samples shear microstructure, from which estimates of the dissipation rate, $\varepsilon$, of turbulent kinetic energy (TKE) were determined (Fer et al. 2014; Palmer et al. 2015). During the OSMOSIS cruise, the glider profiled between the sea surface and $\sim 100$ m depth, capturing 1420 profiles over 9.5 days. A profile was obtained approximately every 10 minutes, with a 20 minute gap for data upload every 10 profiles.

Spikes in the raw OMG microstructure shear data were removed by hand, after which the dissipation was calculated in bins of approximately $1 \mathrm{~m}$ vertical resolution following the methods 
outlined in Merckelbach et al. (2010), Fer et al. (2014) and Palmer et al. (2015). Close to the ocean surface the dissipation estimates can be contaminated by glider motions induced by surface waves. To account for this, the near surface portion of the glider dissipation profiles have been truncated to exclude data from the surface to the deepest of: a) the significant wave height; b) the point where the glider speed drops below one standard deviation from the median (for this deployment); and c) the point where the glider pitch changes by more than one standard deviation from the median value (for this deployment). Typically the cutoff depth is about $5 \mathrm{~m}$.

Conductivity, temperature and depth (CTD) were provided by standard payload sensors (Seabird Electronics) housed in the central section of the OMG and are used to calculate salinity and density. CTD data were collected at $1 \mathrm{~Hz}$ during periods when the MicroRider was operative. Errors in salinity and density may occur due to inconsistencies between temperature and conductivity sensors, which are partly attributable to the physical separation of sensors and different response times, both of which can be simply corrected for. The raw temperature data from the un-pumped CTD sensor was low pass filtered (using a 3rd-order Butterworth filter), subsequently corrections to account for thermal inertia in the conductivity cell were made following the methods of Lueck and Picklo (1990) using modified parameters according to Palmer et al. (2015).

Temperature and salinity were then calibrated against CTD profiles obtained from the ship. Between year days 269 - 270.4, the un-pumped CTD sensor on the glider failed, and for this period temperatures have been obtained from the MicroRider probe. To ensure the MicroRider temperatures were consistent with the CTD temperatures a regression was made between the two instruments when they showed the same structure, between the deep water $(75-100 \mathrm{~m})$ and surface waters $(1-20 m)$, and was used to reconstruct temperature when the CTD failed. It was not possible to reconstruct the missing salinity data. 


\section{b. The TRIAXYS Wave Buoy}

The TRIAXYS directional wave buoy was deployed on the $7^{\text {th }}$ September and recovered on the $27^{\text {th }}$ September 2012, providing spectral energy data from 0 to $0.64 \mathrm{~Hz}$ in frequency bins of $0.01 \mathrm{~Hz}$, with directional-dependence resolved to $3^{\circ}$ divisions, captured every 20 minutes. The Stokes drift, $U_{s 0}$, is the integral of the third moment of the energy spectrum, and is estimated following Webb and Fox-Kemper (2011), namely:

$$
U_{s 0}=\frac{16 \pi^{3}}{g} \int_{0}^{2 \pi} \int_{0}^{\infty}(\cos \theta, \sin \theta) \omega^{3} F^{2}(\omega, \theta) d \omega d \theta
$$

where $\omega$ is the wave frequency and $\theta$ the directional-angle. In practice the wave buoy has a physical cut-off frequency after $0.64 \mathrm{~Hz}$, such that the highest frequency components of the wave field are not resolved. Thus for each time stamp and direction, a best-fit tail is extrapolated from the cut-off frequency using a minus $5^{\text {th }}$ order power law (Phillips 1977). This directional patch for the unresolved tail is added to the resolved spectra and similarly integrated over frequency. Non-directional Stokes drift data used in this study are taken as the absolute values of the total directional Stokes drift, given at 20 minute intervals.

\section{c. Ship Data}

Atmospheric data were sampled throughout the cruise, using the ship's continuous recording instrumentation. Wind speed, direction, atmospheric pressure, air temperature, relative humidity, up-welling and down-welling shortwave irradiances were all measured at a height of $18 \mathrm{~m}$ above mean sea surface. In all cases raw data was recorded at 10s intervals. Quality control, de-spiking and smoothing was applied to all data following Inall and Audsley (2012). The $u$ and $v$ components of the wind were smoothed, and obvious spikes removed manually. The remaining data was then interpolated onto a regular grid and a 120s median smoothing window applied. 
The surface (air) friction velocity was calculated using:

$$
u_{* a}^{2}=C_{D} W_{10}^{2}
$$

where $u_{* a}$ is the friction velocity on the air side of the air-ocean interface. The drag coefficient, $C_{D}$, and $10 \mathrm{~m}$ wind speed, $W_{10}$, were obtained iteratively by applying a log-law boundary layer to adjust for measurement height (Beardsley and Pawlowicz 1999).

The surface buoyancy flux, $B_{0}$, was calculated from the net surface heat flux, $H_{0}$ as:

$$
B_{0}=-C_{T} g \frac{H_{0}}{\rho C_{p}}
$$

where $C_{T}=1.6 \times 10^{-4} \mathrm{~K}^{-1}$ is the thermal expansion coefficient, $g=9.81 \mathrm{~ms}^{-2}$ is gravitational acceleration, $\rho$ is the water density and $C_{p}=3993 \mathrm{Jkg}^{-1} \mathrm{~K}^{-1}$ is the specific heat capacity of water. The net surface heat flux was calculated using:

$$
H_{0}=S W+I R+S H+L E
$$

with shortwave radiation (SW) from the total incident radiation (TIR) sensors on-board, the longwave (IR) radiation obtained from re-analysis data (National Oceanography Centre/University of Southampton, 2008) and the sensible (SH) and latent (LE) heat fluxes obtained using the TOGA COARE 2.0 algorithm (Fairall et al. 1996). To account for shading of the irradiance sensors, values of the TIR were created by taking the maximum value recorded by the port and starboard sensors. Currents were determined with an RDI Ocean Surveyor $75 \mathrm{kHz}$ Vessel Mounted-ADCP, configured to sample over 120 second intervals with 96 bins of $8 \mathrm{~m}$ length, giving a standard deviation of $1.1 \mathrm{cms}^{-1}$. The instrument calibration and calculations of the GPS accuracy are documented in the D381 Cruise report (Allen et al. 2012). During some high wind/wave events data drop-out was apparent in the ADCP current profile data, probably due to cavitation below the ship's hull. This data was identified and masked for any 120 s epoch that was more than $35 \%$ incomplete between 
the surface and $200 \mathrm{~m}$ depth, this systematically removed most of the bad data, however the data around these incidents should be treated with caution.

\section{d. Modelling}

The dissipation rates in the transition layer obtained from the OMG glider, will be compared with a simple parametrisation of the dissipation due to shear production. The parametrisation is based on results from LES, and the derivation of the parametrisation is given in Appendix A.

A simple bulk model of the OSBL is used to determine the inertial currents in the OSBL, and the evolution of the mixed layer depth. The model is described in Appendix B. The thickness of OSBL is assumed to increase through entrainment, with two parametrisations of entrainment considered. The first assumes that entrainment is driven by a combination of convective and Langmuir turbulence (this will be referred to as the Langmuir model). Parametrisation of entrainment due to Langmuir turbulence have been proposed by Grant and Belcher (2009), McWilliams et al. (2013) and $\mathrm{Li}$ and Fox-Kemper (2017). The second parametrisation assumes that entrainment is due to a combination of convective and conventional shear turbulence (referred to as the shear model). The parametrisation for shear turbulence is taken from Grant and Belcher (2009), which is similar to the parametrisation due to $\mathrm{Li}$ and Fox-Kemper (2017).

It is generally thought that Langmuir turbulence is important in the OSBL (McWilliams et al. 1997; D'Asaro 2014), and so in the main part of the paper the results from the Langmuir model will be shown. However, since data on the occurrence of Langmuir turbulence is limited, and there is uncertainty as to when it may be a poor representation of turbulence in the OSBL, the results from the shear model will be considered in section 5 .

The model is forced using ERA-Interim data, that includes wave data. The friction velocity, surface Stokes drift and buoyancy fluxes from ERA-Interim are in very good agreement with the 
estimates obtained from the on-board meteorological measurements and the Stokes drift obtained from the TRIAXYS wave buoy. Differences in the surface fluxes obtained from ERA-Interim and from the ship data contribute to the uncertainties in making comparisons between the model and the observations. In lieu of formal estimates of the surface flux errors, it was decided to use ERA-Interim to force the models, and where necessary, the ship based flux to derive estimates of entrainment fluxes from the observations.

The initial temperature and salinity profiles that are used to initialise the bulk model were obtained from the glider and resolved to a grid of $1 \mathrm{~m}$. These profiles were used to provide the temperature and salinity structure below the OSBL, which was assumed to remain constant through the storm period. The initial depth of the WML was taken to be $35 \mathrm{~m}$, the same as the average mixed layer depth obtained from the glider profiles at the beginning of the storm.

\section{Surface Forcing and the Evolution of the OSBL}

Figures 2(a) and (b) show timeseries of the surface friction velocity, Stokes drift and the surface buoyancy flux obtained from the ship and buoy data over the full 9.5 days of the glider deployment. Time-depth cross sections of temperature and the turbulent dissipation rate are shown in Figs. 2(c) and (d), where the cyan line shows the mixed layer depth determined from the temperature profiles, defined as the level at which the temperature is $0.2^{\circ} \mathrm{C}$ lower than the temperature at a depth of $10 \mathrm{~m}$ (de Boyer Montégut et al. 2004). The blue line shows the depth of the base of the transition layer, which is the depth below the MLD of the deepest isopycnal that lies wholly within one standard deviation of the mean mixed layer depth (see Eq. 1 in Johnston and Rudnick (2009)). This definition assumes that the transition layer thickness is related to the vertical displacements of the MLD and deeper isopycnals. These displacements have been found to be similar to the RMS displacement of a typical open ocean internal-wave spectrum (Johnston and Rudnick 2009; 
Munk 1981) which suggests that internal waves are heaving the MLD and deeper isopycnals into and out of contact with surface-intensified mixing, creating the transition layer defined here.

The first 5 days (days 261 to 266) of the period are characterised by relatively low winds, and an average buoyancy flux which is negative, i.e. the ocean is gaining heat. During this early period the surface buoyancy flux shows a strong diurnal cycle. Although the mixed layer depth, obtained from the temperature profiles, is approximately constant with time, the depth of the OSBL implied by the thickness of the layer in which the dissipation rate is high, shows marked diurnal variations.

During the daytime, large values of TKE dissipation are generally limited to a layer near the surface, that is less than about 10m deep. During the night, large values of TKE dissipation extend down to the stable layer at the base of the WML, and is consistent with the turbulence generated by the loss of buoyancy at the surface. This pattern of a shallow, stable OSBL during the day, followed by a deeper convective OSBL at night is repeated over several days, with the exception of day 263 , when the depth of the OSBL remains large during the day coinciding with elevated winds and reduced buoyancy flux.

Between days 266 and 270, a significant storm passed through the area, with maximum winds speeds reaching $\sim 20 \mathrm{~m} \mathrm{~s}^{-1}$ and significant wave heights of $\sim 6 \mathrm{~m}$. The beginning and end of the storm are marked by the green lines in Fig. 2. During the storm the sensible and latent heat fluxes at the surface increase, with an average buoyancy flux for the period of the storm which is negative, indicating cooling of the surface waters.

Figure 2(c) shows that the stratification at the base of the OSBL weakens during the storm. Temperature profiles obtained during the storm are shown in Fig. 3. During the storm the WML and the transition layer tend to cool, although there is significant variability between the profiles, which is probably associated with submesoscale variations, which are present in the area throughout the 
year (Thompson et al. 2016). Over the period the mixed layer depth increases from about $32 \mathrm{~m}$ to $41 \mathrm{~m}$ while the depth of the base of the TL increases by about $4 \mathrm{~m}$.

Just prior to the start of the storm ( $\sim$ day 266.5) there is a change in salinity of $\sim 0.3 \mathrm{~g} \mathrm{~kg}^{-1}$ below the OSBL (not shown), that coincides with the temperature change at this depth (Fig. 2(c)), however during the storm the changes in salinity are generally small $\left(\sim 0.05 \mathrm{~g} \mathrm{~kg}^{-} 1\right)$. At the start of the storm, Fig. 2(c) shows that there is a warming of the WML, that is accompanied by a reduction in the MLD.

The changes in temperature and salinity at the start of the storm may be due to advection, associated with horizontal changes in temperature, or changes in the position of glider relative to the horizontal gradients (Thompson et al. 2016). However, during the storm the data suggest that it is reasonable to consider that advective processes can be neglected, and that changes in the OSBL are primarily due to the surface forcing.

The track of the glider during the storm is shown in Fig. 1, and is consistent with the presence of inertial oscillations during the storm. The glider track shows clockwise rotations, which have a period of $\sim 14.7$ hours, which is close to the local inertial period of 15.9 hours at the latitude of the PAP site.

Figure 4 compares the velocity predicted by the bulk model (see Appendix B) and observed currents from the ship's ADCP. In the model it is assumed that the current below the OSBL is zero, and so there are no tidal or geostrophic currents. To isolate the wind-driven part of the current in the WML, Fig. 4 show the difference between the ADCP measured currents in the WML and at $49 \mathrm{~m}$, the base of the transition layer.

The model predicts that inertial oscillations grow in amplitude from the start of the storm, and are superimposed on a mean wind-driven current. Towards the end of the storm the amplitude of the inertial oscillations is $\sim 0.1 \mathrm{~ms}^{-1}$. 
The observed north-south component of the current (Figure 4b) shows clear inertial oscillations from day 268, when the ship ADCP data is available. The amplitude of the observed oscillations are similar to those predicted by the model. The presence of oscillations is not as clear in the eastwest component of the current. Because of the problems with the quality of the ADCP data during the storm, it is not clear whether the low amplitude of the oscillations in the east-west component of the current is real. With this caveat, the amplitude and timing of the inertial oscillations obtained from the model appear to be reasonable.

Figure 5 shows a time-depth cross section of the dissipation rate during the storm. The depth of the boundary between the high and low dissipation rate increases through the storm. However, in addition to the increase in the depth of the OSBL, there are also oscillations in the depth of the boundary superimposed on the overall increase.

The depth of the base of the OSBL, obtained from the bulk model, is shown by the dashed line in Fig. 5. The depth of the OSBL from the model increases steadily with time, due to entrainment. The magnitude of the deepening is consistent with the overall increase in the depth of the OSBL implied by the dissipation rate, although the model does not reproduce the higher frequency variation in the depth of the WML shown by the dissipation rate. The high frequency variations in the depth of the WML are probably not due to a stabilising influence surface buoyancy flux during daytime. The effects of stabilising surface fluxes on the depth of the OSBL are not included in the model, since in strong winds these effects should be small. In addition, the shoaling of the boundary layer due to a stabilising surface buoyancy flux would not lead directly to changes in the depth of the stable WML determined from the temperature profiles, apparent in Fig. 2(c) and (d).

Figure 6(a) shows the timeseries of the temperatures obtained from the glider, and the temperatures obtained from the Langmuir model, averaged over the depth of the OSBL. Between days 
265 , just before the start of the storm, and day 270 , at the end of the storm, the upper ocean cools by about $1^{\circ} \mathrm{C}$. However, the cooling rate is not constant with time, and during latter part of day 267 , for example, the near surface temperature actually increases.

From day 268, until the end of the storm, the change in the temperature of the OSBL obtained from the bulk model is similar to the observed change. However, before day 268 the model does not reproduce the variation in the temperature. For example, the rapid cooling observed at the start is not reproduced by the model, and the model does not reproduce the local minimum in the observed temperature on day 267 . These variations in the observed temperature are probably due to the presence of submesoscale variations in temperature (Thompson et al. 2016; Whitt and Taylor 2017).

Figure 6(b) shows an expanded view of the period from day 268 to the end of the storm, when the winds are strongest. During this period the fluctuations in the observed temperatures about the general cooling trend are small, making this a good period to evaluate the heat budget of the OSBL. The observed cooling, estimated from a linear fit to the observed temperatures, is $-0.45 \pm 0.05^{\circ} \mathrm{C}$ (shown by the red line in Fig. 6b). The cooling predicted by the model is $-0.38^{\circ} \mathrm{C}$. The overall heat budget of the OSBL that is implied by the model is therefore consistent with the observed budget.

The cooling in the model is due to the surface heat flux and the entrainment flux. The entrainment flux obtained from the model is $-250 \mathrm{Wm}^{-2}$. This is in reasonable agreement with an entrainment flux of about $-285 \pm 65 \mathrm{Wm}^{-2}$, estimated from the observed cooling, assuming it is only due to the surface and entrainment fluxes. In the Langmuir model it is assumed that entrainment is due to a combination of convective and Langmuir turbulence. During this period, the entrainment flux in the model is primarily due to the Langmuir turbulence, which contributes 
about $-200 \mathrm{Wm}^{-2}$ to the total entrainment. The entrainment flux due to convective turbulence is about $-60 \mathrm{Wm}^{-2}$.

The Langmuir model, described in Appendix B, appears to be able to reproduce the evolution of important features of the OSBL, such as its thickness and cooling, during the storm. The Langmuir model assumes that entrainment is associated with Langmuir turbulence. Results obtained by assuming shear and convective turbulence is responsible for entrainment will be considered in section 5 .

\section{The Transition Layer}

\section{a. The Storm Period}

Figures 7(a) and (b) show the dissipation profiles from the glider for two periods, days 266.25 to 267.8 when the winds are increasing, and days 268.25 to 269.8 when the winds were strongest. The glider profiles have been scaled by $w_{* L}^{3} / h_{\mathrm{ml}}$, where $w_{* L}=\left(u_{* w}^{2} U_{s 0}\right)^{1 / 3}$ is the velocity scale for Langmuir turbulence and $h_{\mathrm{ml}}$ is the depth of the WML (Grant and Belcher 2009). The structure for the OSBL from the dissipation profiles is consistent with the temperature profiles shown in Fig. 3, in that both sets of profiles show that the OSBL has two layers, the well-mixed layer and the stratified transition layer. The thickness of the transition layer appears to decrease between the two periods, due to the deepening of the WML, from $31.5 \mathrm{~m}$ to $36 \mathrm{~m}$, while the depth of the base of the transition layer remains at about $47 \mathrm{~m}$.

A profile of the dissipation rate from one of the LES of Langmuir turbulence used in Grant and Belcher (2009) is also shown in Fig. 7(a) and (b). In the WML the LES profile is in reasonable agreement with the observed profiles. The LES dissipation rate profile decreases more rapidly with depth in the transition zone than the observed dissipation, although this difference is less marked 
in the second period, due to the reduction in the thickness of the transition layer. The reduction in the thickness of the transition layer happens because, while the base of the well-mixed layer deepens, the depth of the base of the transition layer remains approximately constant. However, the thickness of the transition layer remains larger than the thickness of the transition layer in the LES profiles. Below the transition layer the observed dissipation rates are much larger than from the LES. This is because the dissipation rates below the transition layer in the real ocean are generally less than the noise level of the microstructure probe.

Skyllingstad et al. (2000) and Grant and Belcher (2011) have used LES to study the development of shear layers at the base of the WML. Skyllingstad et al. (2000) considered two cases. In the first, the surface stress rotated at the inertial period, and remained aligned with the current direction. Large shears developed across the TL, which increased in thickness. In the second case, the direction of the surface stress was kept constant, and the direction of the currents in the OSBL rotated relative to the surface stress. The shear and production of TKE at the base of the WML were much smaller than in the first case. The storm considered in this study corresponds to the second (non-resonant) case in Skyllingstad et al. (2000), since the winds associated with the present storm did not rotate at the inertial frequency.

To make a link between the inertial shear and the turbulence in the transition layer, a comparison is made with a simple parametrisation of the maximum dissipation rate due to shear production at the base of the WML and the dissipation rates from the glider. This parametrisation is tuned using the LES (to determine the coefficients alpha and beta) which is described in Appendix A, and is given by, 


$$
D=0.3 \exp \left(-4.5 \frac{f h_{\mathrm{bl}}}{u_{* w}}\right)\left(\operatorname{MAX}\left\{\frac{u_{* w}^{2}\left(\langle\mathbf{U}\rangle_{m l}-\mathbf{U}_{e x t}\right)}{h_{\mathrm{bl}}}, 0\right\}+\beta M A X\left\{f U_{s 0} \delta \frac{\left(\langle\mathbf{V}\rangle_{m l}-\mathbf{V}_{e x t}\right)}{\Delta h}, 0\right\}\right)
$$

where $D$ is the maximum in the dissipation rate, $\langle\mathbf{U}\rangle_{m l}$ and $\langle\mathbf{V}\rangle_{m l}$ are the components of the current parallel and perpendicular to the direction of the surface stress, averaged over the depth of the WML, $\mathbf{U}_{\text {ext }}$ and $\mathbf{V}_{\text {ext }}$ are the current components below the OSBL, $u_{* w}$ is the water-side surface friction velocity, $h_{\mathrm{bl}}$ is the depth of the boundary layer. The surface friction velocity is defined by, $u_{* w}^{2}=-\overline{\mathbf{u}^{\prime} \mathbf{w}^{\prime}} 0$, where $\overline{\mathbf{u}^{\prime} \mathbf{w}^{\prime}}$ is the surface value of the momentum flux, $U_{s 0}$ is the surface Stokes drift, $\delta$ is the Stokes penetration depth and $f$ is the Coriolis parameter.

The first term in the brackets, after the exponential function, on the right-hand side of Eq. 5 represents the dissipation due to shear production by the current shear in the direction of the surface stress, and is the same as the parametrisation given by Grant and Belcher (2011) for the resonant conditions. The second term represents the shear production that is associated with the current shear perpendicular to the surface stress. Although $\overline{v^{\prime} w^{\prime}}$ at the surface is zero, it increases rapidly with depth, and reaches maximum value just below the surface. The gradient of $\overline{\mathbf{v}^{\prime} \mathbf{w}^{\prime}}$ above the maximum is assumed to be balanced by the Stokes-Coriolis force (Polton et al. 2005). The non-zero value of $\overline{\mathbf{v}^{\prime} \mathbf{w}^{\prime}}$ within the OSBL leads to the production of TKE from the lateral shear at the base of the WML. Each of the components of the shear production must be greater than zero, i.e. the shear terms in the TKE budget do not act as a sinks for the TKE.

Figure 8(a)-(d) shows a comparison of timeseries of observed and predicted dissipation rate at different depths relative to the base of the WML. Figure 8(a) shows the dissipation rate $5 \mathrm{~m}$ above the base of the WML, i.e. within the lower part of the WML. The dissipation rate at this depth 
gradually increases with time, as the surface wind increases. The curve in Fig. 8(a) shows the dissipation rate calculated from,

$$
\varepsilon=0.05 \frac{w_{* L}^{3}}{h_{\mathrm{ml}}}+0.4 B_{0}
$$

where the first term on the right-hand side is the dissipation rate due to Langmuir turbulence at the base of the WML. Grant and Belcher (2009) show for Langmuir turbulence the dissipation rate around the base of the WML is $\sim 0.1 w_{* L}^{3} / h_{\mathrm{ml}}$, and the lower value for the coefficient in Eq. 6 reflects the rapid variations of the dissipation rate with depth around the base of the WML. The second term is the dissipation rate due to the convective turbulence, which is assumed to be constant with depth (Lombardo and Gregg 1989). There may be periodic variations in the dissipation rate at this level, but if they are present they are not clear.

The magnitude of the dissipation rate in the TL decreases with increasing depth (see Fig 7). In addition, in the layer $5 \mathrm{~m}$ to $10 \mathrm{~m}$ below the base of the WML, the magnitude of the dissipation rate shows clear oscillations in time, the amplitude of the oscillations decreasing with depth. The black curves in Figs. 8(b)-(d) show the dissipation rates obtained from Eq. 5, assuming that the thickness of transition layer is $10 \mathrm{~m}$. The decrease in the amplitude of the oscillation in the dissipation rate with depth below the WML, is consistent with the amplitude of the oscillations going to zero around the base of the TL. Since Eq. 5 gives the dissipation rate at the base of the WML, to capture the decrease in the dissipation rate within the TL, the values obtained from Eq. 5 have been multiplied by 1.0, 0.5 and 0.25 in Figs. 8(b) to (d). The magnitude of these factors is reasonable given that the dissipation rate tend to zero at the base of the TL, but having to use them means that a precise value of the coefficient in Eq. 5 can not be assessed using the data. A more complete parametrisation of the dissipation rate due to shear production in the TL would specify the depth dependence of the dissipation rate in the TL. 
From the start of the storm, until the middle of day 268 , the predicted dissipation rates from

Eq. 5 are in good agreement with the observations. At $5 \mathrm{~m}$ below the base of the WML, the observations show some high dissipation rates, which may reflect the presence of the relative large dissipation rates just above this depth. After day 268.5, the dissipation rates calculated from Eq. 5 are about a factor of two larger than the observed dissipation rates, as shown by the red curves which show results from Eq. 5, multiplied by the factors given above and divided by two. The red curves match the observations during this period.

The bulk model does not give any information on the structure of the transition layer, and in estimating the dissipation rates from the parametrisation the thickness of the transition layer has been assumed to be a constant 10 metres. However, Figs. 7(a) and (b) show that the thickness of the transition layer decreases through the storm, and this may explain why, when matched to the dissipation rate before day 268.5 , the parametrisation overestimates the dissipation rate after day 268.5. A more sophisticated model and parametrisation would be needed to predict the evolution of the structure of the TL.

Grant and Belcher (2011) found that the magnitude of the buoyancy flux at the base of the WML, due to the shear production of turbulence, was $\sim 33 \%$ of the dissipation rate obtained from Eq 5. Using this with Eq. 5 suggests that the heat flux at the base of the WML due to shear production between days 268.25 and 269.8 is $\sim-30 \mathrm{Wm}^{-2}$. This is about $12 \%$ of the entrainment flux attributed to convective and Langmuir turbulence in the Langmuir model. This implies that for this storm the effects of shear turbulence on the evolution of the OSBL were small.

This analysis shows that (i) the evolution of the dissipation rate within the TL follows a different behaviour to the evolution of the dissipation rate within the ML, (ii) that the oscillations in the dissipation rate are coherent through the TL, (iii) that the period of the oscillations is close to the local inertial period, about 15.9hours. 


\section{b. Post Storm Shear Spikes}

Burchard and Rippeth (2009) observed that enhanced turbulence was linked to alignment of the shear across the base of the OSBL with the direction of the surface wind. They called these periods of enhanced shear, shear spikes. The observations used by Burchard and Rippeth (2009) were obtained on the continental shelf, with a water depth of $\sim 100 \mathrm{~m}$, where the currents were affected by tides. Brannigan et al. (2013) showed that shear spikes were present in the open ocean, although were unable to show that they were associated with periods of enhanced turbulent mixing. The models of Burchard and Rippeth (2009) and Brannigan et al. (2013) predict the occurance of periods of enhanced shear, and so increased likelihood of shear instability. However, they do not predict the dissipation rate associated with the shear spikes.

Rumyantseva et al. (2015) observed similar shear spikes, with enhanced turbulence, at the PAP site on day 271 , after the storm and shortly after the microstructure glider had been recovered. The surface winds were much lighter on day 271 than during the storm, but the currents continued to show the large amplitude inertial oscillations generated by the storm. Rumyantseva et al. (2015) measured the dissipation rate using an MSS90 microstructure profiler, and found that dissipation rates increased by over an order of magnitude during the shear spikes, reaching a magnitude of $\sim 10^{-7} \mathrm{~W} \mathrm{~kg}^{-1}$

The model simulation was continued to cover the period of the observations of Rumyantseva et al. (2015) (not shown). The dissipation rates estimated from Eq. 5 for day 271 were much smaller than the observed dissipation rates during the shear spikes. The temperature-depth cross section given in Rumyantseva et al. (2015) suggests that the thickness of the pycnocline is smaller than it was during the storm, and that the temperature change across the pycnocline is smaller. The appearance of spikes in the dissipation rate during this period may be due to these changes, 
although they don't explain why Eq. 5 doesn't apply during this period. This point will be considered further in section 5 .

\section{Discussion}

We have presented measurements of the dissipation rate in the North Eastern Atlantic which were obtained using a microstructure glider. During the period when the glider was deployed a storm passed over the area, and the data from the microstructure glider showed that there were oscillations in the dissipation rate in the transition layer at the base of the OSBL. The period of the oscillations was close to the local inertial period. The main aim of the study has been to compare the behaviour of the dissipation rate in the transition layer with a simple parametrisation of the dissipation associated with the production of TKE due to inertial oscillations in the current shear. The parametrisation was derived using results obtained from LES.

Reliable measurements of the current shear were not available, and to determine the current shear needed by the parametrisation, a simple bulk model (described in Appendix B) was used (Niiler and Kraus 1977). This model included a parametrisation of entrainment, which for the results shown in the previous sections assumed that turbulence in the OSBL was due to a combination of convective and Langmuir turbulence. In addition to predicting the currents during the storm, the model also predicted the evolution of the temperature and the depth of the base of the OSBL, which could be compared with the observations.

The change in the depth of the OSBL base, and the cooling of the OSBL obtained from the model were in reasonable agreement with the observed changes (Figs. 5 and 6). The agreement suggests that the assumption that Langmuir turbulence was present in the OSBL, and the parametrisation of entrainment by Langmuir turbulence obtained from LES are reasonable. 
However, variations in the near surface temperature, which are probably due to the presence of submesoscale variability (Thompson et al. 2016), make it difficult to conclude from this comparison that the Langmuir turbulence must be present. In particular, the observed cooling of the OSBL may have been affected by advection, and it is possible that the observations are consistent with other assumptions about entrainment. It is useful to compare the results from the Langmuir model with results obtained from a model in which entrainment is assumed to be due to conventional shear driven turbulence.

Over the last 1.5 days of the storm the cooling from the Langmuir model is $-0.38^{\circ} \mathrm{C}$, which is similar to the observed cooling of $-0.45 \pm 0.05^{\circ} \mathrm{C}$. The model suggests that entrainment is a significant term in the heat budget of the OSBL, and the agreement between the modelled and observed cooling depends on the parametrisation of entrainment.

The parametrisation for entrainment can be changed for one in which entrainment is assumed to be driven by convective and conventional shear turbulence (see Appenix B). Using this model, the cooling obtained over the same period is $-0.31{ }^{\circ} \mathrm{C}$. The reduction in the cooling is due to the parameterised entrainment flux being smaller than that in the Langmuir model. The entrainment flux due to shear turbulence, obtained from the shear model, is $\sim-116 \mathrm{Wm}^{-2}$ compared to $\sim-250 \mathrm{Wm}^{-2}$ from the Langmuir model. The cooling from the shear model is just about consistent with the observations, particularly if the observed cooling is influenced by more than just the surface and entrainment fluxes. In addition to the uncertainties associated with the observations, the constants used in the parametrisation are derived from LES, and may differ from values that might be obtained from direct observations. From this comparison it is not possible to conclude that the Langmuir model is better than the shear model, although the Langmuir model gives reasonable results. 
The change in the thickness of the OSBL during the storm obtained from the models also depends on the parametrisation of entrainment. For the Langmuir model the change in the thickness of the OSBL over the storm is about $8.8 \mathrm{~m}$, while for the shear model the change $5.4 \mathrm{~m}$. Figure 5 shows that there is significant variability in the depth of the OSBL base, in addition to the overall increase in the thickness. This variability may be associated with the submesoscale variability in the area of the observations. The presence of this variability in the thickness OSBL in the observations means it is not possible conclude that the Langmuir model is better than shear model, although again the results from the Langmuir model are reasonable.

What might be needed to come to a more definite conclusion? The differences between the two models used here increase with time, and in a storm of longer duration it is possible that the differences in the cooling and the change in the depth of the OSBL might become large enough for a more definite conclusion to be reached. With glider technology it should be possible to obtain more data during storms to help confirm the general presence of Langmuir turbulence in such situations, and the usefulness of LES in developing parametrisations, through studies such as this.

The agreement between the non-dimensional dissipation rates within the OSBL with the results from LES of Langmuir turbulence, given by Grant and Belcher (2009), cannot be used as support for the presence of Langmuir turbulence during the storm. The reason is that, when scaled with $u_{* w}^{3} / h_{\mathrm{ml}}$, Grant and Belcher (2009) showed that when the Langmuir number is $\approx 0.3$, the nondimensional profiles agree with profiles of LES of conventional shear turbulence. Sutherland et al. (2014) have presented observations which suggest that the dependence of the non-dimensional dissipation rate on the Langmuir number is consistent with that found by Grant and Belcher (2009), but the variation in Langmuir number in the present data is not sufficient to confirm this.

The parametrisation of the dissipation rate given in Eq. 5 was obtained by assuming the base of the TL corresponds to the base of the OSBL, and that the profiles of $\overline{\mathbf{u}^{\prime} \mathbf{w}^{\prime}}$ and $\overline{\mathbf{v}^{\prime} \mathbf{w}^{\prime}}$ go to 
zero at the base of the OSBL/TL. The dissipation rate in the TL varies with time because of the interaction between the boundary layer stresses and the time varying shear across the TL due to the inertial oscillations of the currents within the OSBL. In the LES, which were used to develop the parametrisation of the dissipation rate (Eq. 5), the bulk Richardson number for the TL was between 0.2 and 0.4 (Grant and Belcher 2011), and the thickness of the shear layer increased with time. However, during the storm, the bulk Richardson number of the TL was $\sim 4$ when the turbulence dissipation was a maximum, and rather than increasing with time, the thickness of the transition layer decreased with time (see Fig. 7). Despite these differences in the stability of the TL, the comparison shown in Fig. 8 suggests that the Eq. 5 is a reasonable parametrisation, although it is not obvious why this should be.

A possible reason why Eq. 5 works during the storm is that the TL is not an isolated shear layer, but is connected to the well-mixed region of the OSBL through the transport of turbulent kinetic energy into the upper part of the TL. Studies of entrainment in the sheared convective atmospheric boundary layer show that shear production of turbulence in the inversion can occur for gradient Richardson number significantly above 1/3 (Haghshenas and Mellado 2019), similar to the situation during the storm. Haghshenas and Mellado (2019) found that as the shear increases, the gradient Richardson number tends to a value of about $1 / 3$, similar to the values in the LES (Grant and Belcher 2011). While the present situation is not directly comparable to entrainment in the atmospheric boundary layer, the interaction between the well-mixed layer and the transition layer through the transport of TKE may help explain why the results obtained with Eq. 5 are reasonable. Further studies are needed to improve our understanding of processes in the transition layer.

In this study the parametrisation for the dissipation in the TL has only used diagnostically, and the effects of the shear generated turbulence were not included in the bulk model. This is 
reasonable since the shear production of turbulent kinetic energy during this storm was relatively low, but in a more complete model, in which the effects of the shear production of TKE are included, a representation of the evolution of the transition layer may be possible. In particular, the evolution of the depth of the transition layer, which was simply specified in the present study, would need to be modelled.

The microstructure glider was recovered after the end of the storm, but further measurements of the dissipation were obtained after the storm using a profiler deployed from the Discovery (Rumyantseva et al. 2015), finding evidence of shear spikes (Burchard and Rippeth 2009; Lenn et al. 2011; Brannigan et al. 2013; Lincoln et al. 2016). Shear spikes are associated with the generation of turbulence within the transition layer, as a result of surface wind and current shear alignment which produces enhanced shear. The dissipation rate during the shear spikes was $\approx$ $10^{-7} \mathrm{~W} \mathrm{~kg}^{-1}$, which is similar to the peak dissipation rates due to shear production that were observed in the TL during the storm. However, during the post-storm period the surface winds were much lighter than winds during the storm, and the dissipation rates implied by the present model are negligible, due to the dependence of the dissipation rate on $f h_{\mathrm{bl}} / u_{* w}$.

The rate of work by the surface stress acting on the inertial currents in the OSBL is $u_{* w}^{2} U / h_{\mathrm{bl}}$, where $U$ is the current parallel to the surface stress, averaged over the depth of the OSBL, (Grant and Belcher 2011). This can be thought of as the divergence of a flux of mean kinetic energy, where the surface flux is $u_{* w}^{2} U$. For the post-storm period, currents from the model give $u_{* w}^{2} U / h_{\mathrm{bl}} \approx 10^{-7}$ $\mathrm{W} \mathrm{kg}{ }^{-1}$. The coincidence in the magnitude of the dissipation rate during the shear spikes and the rate at which work is being done by the surface stress acting on the inertial currents suggests that the turbulence associated with shear spikes arises directly from the breakdown of the shear at the base of the OSBL. Since the turbulence is assumed to occur because of the work done on the mean flow by the surface stress, there is no contribution to the production of TKE from the component 
of the current that is perpendicular to the surface stress. This is in contrast to the parametrisation given in Eq. 5 where the production of TKE by the lateral shear is assumed to occur, as the steadystate momentum balance of the OSBL implies $\overline{\mathbf{v}^{\prime} \mathbf{w}^{\prime}}$ is not zero below the surface. The changes in the properties of the pycnocline that occurred after the storm, which should have reduced the bulk Richardson number, would make the generation of turbulence from the simple breakdown of the shear possible.

The data presented in this study has been analysed using a one-dimensional framework, and any effects due to submesoscale processes have been neglected. Whitt and Taylor (2017) have recently presented results from a large-domain LES (horizontal domain 1.9x1.9 km) of the storm in this study. By coincidence their domain size was comparable to the diameter of the circular path taken by the glider during the storm (see Fig 1a).

This simulation shows submesoscale features, with scales of order $1 \mathrm{~km}$, develop during the storm, and help to maintain stable stratification within the mixed layer. The variability in the temperatures measured by the glider, shown in Fig. 6, may be due to this submesoscale variability. Whitt and Taylor (2017) also found that turbulence levels showed significant horizontal variability that was related to the submesoscale variability in the stratification. Given these results, it is reasonable to ask if the one-dimensional approach used here is valid.

The results of this study suggest, that despite the presence of submesoscale variability, the onedimensional assumption is reasonable. Dissipation rates within the bulk of the OSBL are consistent with scaling results from LES. The bulk model produced reasonable estimates for the cooling of the OSBL, and the increase in the thickness of the OSBL. While the presence of submesoscale variability may have made it difficult to decide which form of turbulence was present in the WML (Langmuir or shear), the evolution of the OSBL could be described reasonably well using a onedimensional framework. 


\section{Summary}

This paper has used observations from a microstructure glider, together with a simple bulk model of the OSBL and dissipation in the transition layer, to help understand the turbulence and evolution of the OSBL during a storm. The key results from this study are,

- The OSBL has a two layer structure, a well mixed layer separated from the deeper water by a stratified transition layer. The flow in the transition layer is turbulent, with the dissipation rate showing periodic variations, with a period close to the local inertial period ( $\sim 15.9$ hours).

- A parametrisation of the dissipation rate due to shear turbulence was developed using results from LES. The dissipation rates obtained from the parametrisation were in reasonable agreement with the dissipation rates obtained by the microstructure glider in the TL. The Richardson number for the TL was about 4, suggesting that it was too stable for shear turbulence to develop. It is possible that the transport of TKE from the WML into the TL plays a role.

- The evolution of the thickness of the OSBL and its heat budget were obtained using a simple bulk model in which entrainment was assumed to be due to Langmuir turbulence. The parametrisation of entrainment due to Langmuir turbulence was obtained from LES. The model results suggest that cooling of the OSBL due to entrainment was significant. Although the observations are consistent with this model, and the presence of Langmuir turbulence, the duration of the storm was not long enough conclude that this model was better than one in which entrainment is assumed to be due to conventional shear turbulence.

- The parametrisation of the dissipation rate developed in this study did not predict the occurrence of dissipation due to shear spikes after the storm. It is not clear why, but it was noted that the observed dissipation rate during the shear spikes was comparable to the rate of work 
done by the surface stress on the mean currents in the OSBL, and that changes to the pycnocline probably made the breakdown of the shear likely. However, further work is needed to understand the generation of turbulence associated with shear spikes.

- The parametrisations for entrainment in the bulk model were obtained from LES. Since the constants in the bulk model were obtained from the LES it was not necessary to tune the model to other observations, and the results from the study provide an example of the usefulness of LES in developing parametrisations.

Acknowledgments. We are grateful to the engineers and scientists at NMFSS, captain and crew of the RRS Discovery, and numerous scientists and technicians that helped during deployment and recovery of the glider and wave buoy and aboard the RSS cruise D381b. All data are archived at the British Oceanographic Data Centre. This research was funded by grants from the Natural Environmental Research Council OSMOSIS (NE/1020083/1) and FASTNEt (NE/1030224/1).

\section{APPENDIX A}

\section{Parametrisation of shear production at the base of the OSBL}

In this appendix a parametrisation of the shear production of TKE at the base of the OSBL is developed. The parametrisation is based on the work of Grant and Belcher (2011), which is extended to include the effects of inertial oscillations in the OSBL. Grant and Belcher (2011) only considered the case where the direction of the surface stress and the currents in the OSBL were aligned and constant in time (which was approximated by setting the Coriolis parameter to zero). They developed the following parametrisation for the generation of turbulence kinetic energy (TKE) by the shear, 


$$
S_{U}=-\left.\overline{\mathbf{u}^{\prime} \mathbf{w}^{\prime}}{ }_{m l} \frac{\partial \mathbf{U}}{\partial z}\right|_{m l} \sim \frac{u_{* w}^{2}\left(\langle\mathbf{U}\rangle_{m l}-\mathbf{U}_{e x t}\right)}{h_{\mathrm{bl}}}
$$

where, $S_{U}$ is the shear production, $\mathbf{U}$ is the component of the current in the direction of the surface stress, $\partial \mathbf{U} /\left.\partial z\right|_{m l}$ is the shear at the base of the well-mixed layer, $\overline{\mathbf{u}^{\prime} \mathbf{w}^{\prime}}{ }_{m l}$ is the turbulent momentum flux at the base of the well-mixed layer, $u_{* w}$ is the surface friction velocity of water, $\langle\mathbf{U}\rangle_{m l}$ is the average velocity in the well-mixed layer, $h_{\mathrm{bl}}$ is the depth of the boundary layer and $\mathbf{U}_{e x t}$ is the current velocity at the base of the boundary layer. The surface friction velocity is defined as, $u_{* w}^{2}=-\overline{\mathbf{u}^{\prime} \mathbf{w}^{\prime}}{ }_{0}$, where $\overline{\overline{\mathbf{u}}^{\prime} \mathbf{w}^{\prime}}{ }_{0}$ is the surface value of the momentum flux.

When the currents and surface stress rotate, the total shear production is,

$$
S=-\left.\overline{\mathbf{u}^{\prime} \mathbf{w}^{\prime}} m l \frac{\partial \mathbf{U}}{\partial z}\right|_{m l}-\left.\overline{\mathbf{v}^{\prime} \mathbf{w}^{\prime}} m l \frac{\partial \mathbf{V}}{\partial z}\right|_{m l}
$$

where, $S$ is the total shear production, $\mathbf{V}$ is the component of the current perpendicular to the surface stress, $\overline{\overline{\mathbf{v}}^{\prime} \mathbf{w}^{\prime}}$ ml is the lateral component of the momentum flux, at the base of the well-mixed layer, (and $\overline{\mathbf{u}^{\prime} \mathbf{w}^{\prime}} m l$ is the component in the direction of the surface momentum flux) and $\partial \mathbf{V} /\left.\partial z\right|_{m l}$ is the shear in the lateral component of the current, at the base of the well-mixed layer.

Following Grant and Belcher (2011), ${\overline{\mathbf{u}^{\prime} \mathbf{w}^{\prime}}}_{m l}$ is parameterised as $\left.-u_{* w}^{2}\left(1-h_{\mathrm{ml}} / h_{\mathrm{bl}}\right)\right)$, where $h_{\mathrm{ml}}$ is the depth of the mixed layer (which for the LES results is defined as the level of the minimum buoyancy flux $)$, and $\partial \mathbf{U} /\left.\partial z\right|_{m l} \propto\left(\langle\mathbf{U}\rangle_{m l}-\mathbf{U}_{e x t}\right) /\left(h_{\mathrm{bl}}-h_{\mathrm{ml}}\right)$, so that the first term of Eq. A2 is given by Eq. A1.

At the surface, $\overline{\mathbf{v}^{\prime} \mathbf{w}^{\prime}} 0=0$, but within the WML $\overline{\mathbf{v}^{\prime} \mathbf{w}^{\prime}}$ is positive (in the northern hemisphere), with a maximum in the lower part of the OSBL (Zikanov et al. 2003). The maximum value of $\overline{\mathbf{v}^{\prime} \mathbf{w}^{\prime}}$ can be estimated by considering the balance between the Coriolis force on the near surface drift and stress gradient. The magnitude of the near surface drift is about $3 \%$ of the windspeed (Wu 
1983), and in the open ocean is mainly due to the Stokes drift that is associated with the surface waves (Wu 1983). Taking the depth of the maximum in $\overline{\mathbf{v}^{\prime} \mathbf{w}^{\prime}}$ to be $\sim \delta$, where $\delta$ is the Stokes penetration depth, the balance between the stress gradient and the Stokes-Coriolis force gives,

$$
{\overline{\mathbf{v}^{\prime} \mathbf{w}^{\prime}}}_{m l} \sim f u_{s 0} \delta
$$

where $f$ is the Coriolis parameter, $u_{s 0}$ is the surface Stokes drift.

Using Eq. A3 the shear production associated with the velocity component perpendicular to the surface stress can be parameterised as,

$$
S_{v}=-\alpha \beta f u_{s 0} \delta \frac{\left(\langle\mathbf{V}\rangle_{m l}-\mathbf{V}_{e x t}\right)}{\Delta h}
$$

where $\langle\mathbf{V}\rangle_{m l}$ is the average of $\mathbf{V}$ over the well-mixed layer, $\mathbf{V}_{e x t}$ is $\mathbf{V}$ at the base of the OSBL, $\Delta h$ is the thickness of the pycnocline and $\beta$ is a coefficient.

The total shear production is the sum of $S_{u}$ from Eq. A1 and $S_{v}$ from Eq. A4, and is given by,

$$
S=\alpha\left(\operatorname{MAX}\left\{\frac{u_{* w}^{2}\left(\langle\mathbf{U}\rangle_{m l}-\mathbf{U}_{e x t}\right)}{h_{\mathrm{bl}}}, 0\right\}+\beta M A X\left\{f U_{s 0} \delta \frac{\left(\langle\mathbf{V}\rangle_{m l}-\mathbf{V}_{e x t}\right)}{\Delta h}, 0\right\}\right)
$$

The results from the LES are consistent with the shear production associated with each of the velocity components having to be greater than zero, which is represented in Eq. A5 by the $M A X$ functions.

Figure A1(a) shows the timeseries of maximum shear production from the LES, compared to the parameterised shear production determined from from Eq. A5. The time in Fig. A1 has been normalised by the inertial period, $T_{I}=2 \pi / f$. The LES used to obtain the estimates of the shear production was the same as the simulations described in Grant and Belcher (2011), but with the Coriolis parameter set to $0.25 \times 10^{-4} \mathrm{~s}^{-1}$. The averages over the well-mixed layer, the thicknesses 
of the well-mixed layer and the shear layer that are needed to calculate the shear production from Eq. A5 were also determined from the LES for this comparison.

For the first $0.4 T_{I}$ the shear production from the LES is large and approximately constant. From $0.4 T_{I}$ to $0.7 T_{I}$ the shear production decreases, becoming constant after $0.7 T_{I}$. Before $0.7 T_{I}$, reasonable agreement between the shear production obtained from Eq. A5, and the shear production from the LES, is obtained with $\alpha=0.2$ and $\beta=1.5$.

Before $0.7 T_{I}$ the time variation on the shear production is associated with the rotation of of the inertial current with respect to the surface stress. After $0.7 T_{I}$ the shear production associated with the inertial shear is zero, and the shear production is due to current shear in the well mixed layer that is associated with Langmuir turbulence (Grant and Belcher 2009).

The value of $\alpha=0.2$ in the parametrisation of the shear production is smaller than $\alpha=0.4$ obtained by Grant and Belcher (2011), and suggests that $\alpha$ is a function of a non-dimensional parameter. The most obvious candidate for this non-dimensional parameter is $f h_{\mathrm{bl}} / u_{* w}$, the ratio of the depth of the OSBL to the Ekman depth, $u_{* w} / f$.

Figure A1(b) shows the values of $\alpha$ obtained by Grant and Belcher (2011), the present value and the value obtained from a third LES with $f=0.5 \times 10^{-4} \mathrm{~s}^{-1}$, as a function of $f h_{\mathrm{bl}} / u_{* w}$. The parameter $\alpha$ decreases as $f h_{\mathrm{bl}} / u_{* w}$ increases. The curve in Fig. 6 shows $\alpha=0.4 \exp \left(-4.5 f h_{\mathrm{bl}} / u_{* w}\right)$, and is used as an approximate parametrisation for $\alpha$ in the main text.

The observations used in the present study are of the dissipation rate, $\varepsilon$ rather than shear production. Grant and Belcher (2011) found that the dissipation was about $75 \%$ of the shear production. Assuming that this holds when the Coriolis parameter is not zero, the parametrisation of the dissipation rate is, 
$D=0.3 \exp \left(-4.5 f h_{\mathrm{bl}} / u_{* w}\right)\left(M A X\left\{\frac{u_{* w}^{2}\left(\langle\mathbf{U}\rangle_{m l}-\mathbf{U}_{e x t}\right)}{h_{\mathrm{bl}}}, 0\right\}+\beta M A X\left\{f U_{s 0} \delta \frac{\left(\langle\mathbf{V}\rangle_{m l}-\mathbf{V}_{e x t}\right)}{\Delta h}, 0\right\}\right)$

\section{APPENDIX B}

\section{The bulk model}

In this appendix we describe the simple bulk model of the OSBL that is used in the body of the paper to understand the evolution of the OSBL shown in the observations. The inertial shear needed to estimate the dissipation is obtained using a bulk model of the OSBL. Following Niiler and Kraus (1977), this model predicts the time evolution of the buoyancy, and components of the current averaged over the OSBL. From the observations, the dissipation rate at the base of the OSBL is small, and so the model does not include the effects of shear turbulence on the evolution of the OSBL. The equations for the currents and buoyancy are,

$$
\begin{aligned}
& \frac{\partial\langle U\rangle_{m l}}{\partial t}=-\frac{\overline{u^{\prime} w_{0}^{\prime}}}{h_{\mathrm{bl}}}-\frac{\left(\langle U\rangle_{m l}-U_{e x t}\right)}{h_{\mathrm{bl}}} \frac{\partial h_{\mathrm{bl}}}{\partial t} \\
& \frac{\partial\langle V\rangle_{m l}}{\partial t}=-\frac{\overline{v^{\prime} w^{\prime}}}{h_{\mathrm{bl}}}-\frac{\left(\langle V\rangle_{m l}-V_{e x t}\right)}{h_{\mathrm{bl}}} \frac{\partial h_{\mathrm{bl}}}{\partial t} \\
& \frac{\partial\langle B\rangle_{m l}}{\partial t}=\frac{\left(\overline{w^{\prime} b^{\prime}}{ }_{e n t}-\overline{w^{\prime} b_{0}^{\prime}}\right)}{h_{\mathrm{bl}}}
\end{aligned}
$$

where $\overline{u^{\prime} w^{\prime}} 0, \overline{v^{\prime} w^{\prime}} 0$ and $\overline{w^{\prime} b^{\prime}}{ }_{0}$ are the surface momentum and buoyancy fluxes and $\overline{w^{\prime} b^{\prime}}$ ent is the buoyancy flux due to entrainment. In Eqs B1 and B2, the components of the current and turbulent fluxes are relative to a fixed, geographic frame.

Following Grant and Belcher (2009), the entrainment buoyancy flux is parametrised as, 


$$
{\overline{w^{\prime} b^{\prime}}}_{\text {ent }}=-0.2{\overline{w^{\prime} b^{\prime}}}_{0}-0.033 \frac{w_{* L}^{3}}{h_{\mathrm{bl}}}
$$

where the first term on the right-hand side is the entrainment flux associated with the forcing by the surface buoyancy flux and the second term represents entrainment due to Langmuir turbulence. In Eq. B4 it has been assumed that the total entrainment due to the combination of convective and Langmuir turbulence is just the sum of the individual entrainment fluxes.

From the results in Grant and Belcher (2009), the entrainment flux due to shear turbulence can be parametrized as,

$$
{\overline{w^{\prime} b^{\prime}}}_{\text {ent }}=-0.2{\overline{w^{\prime} b^{\prime}}}_{0}-0.15 \frac{u_{* w}^{3}}{h_{\mathrm{bl}}}
$$

The equation for the depth of the base of the OSBL is,

$$
\frac{\partial h_{\mathrm{bl}}}{\partial t}=-\frac{{\overline{w^{\prime} b^{\prime}}}_{e n t}}{\Delta B}
$$

where, $\Delta B=\langle B\rangle_{m l}-B_{\text {ext }}$

Equation B6 is describes the evolution of the depth of an entraining boundary layer in mixedlayer models (Niiler and Kraus 1977). The effects of shear turbulence are expected to be small for this storm, and so the effects of current shear on the depth of the OSBL have not been included in Eq's. B4 \& B6. Given the strong winds during the storm, the shortwave irradiance was not large enough to lead to the formation of a shallow, stable boundary layer, and the model only considers the evolution of the depth of the OSBL due to entrainment.

\section{References}

Aijaz, S., M. Ghantous, A. V. Babanin, I. Ginis, B. Thomas, and G. Wake, 2017: Nonbreaking wave-induced mixing in upper ocean during tropical cyclones using coupled hurricane- 
ocean-wave modeling. Journal of Geophysical Research-Oceans, 122, 3939-3963, doi:10.1002/ 2016JC012264.

Alford, M. H., 2003: Improved global maps and 54-year history of wind-work on ocean inertial motions. Geophysical Research Letters, 30 (8), 1424, doi:10.1029/2003GL018543.

Allen, J. T., A. Naveira-Garabato, and A. Forryan, 2012: Cruise Report: RSS Discovery Cruise 381. Tech. rep., National Oceanography Centre, Southampton, England, 1-196 pp.

Beardsley, B., and R. Pawlowicz, 1999: Air-Sea Matlab Toolbox. Woods Hole Oceanographic Institution.

Belcher, S. E., and Coauthors, 2012: A global perspective on Langmuir turbulence in the ocean surface boundary layer. Geophysical Research Letters, 39 (17), 1-9, doi:10.1029/ 2012GL052932.

Brannigan, L., Y.-D. Lenn, T. P. Rippeth, E. McDonagh, T. K. Chereskin, and J. Sprintall, 2013: Shear at the Base of the Oceanic Mixed Layer Generated by Wind Shear Alignment. Journal of Physical Oceanography, 43 (8), 1798-1810, doi:10.1175/JPO-D-12-0104.1.

Burchard, H., and T. P. Rippeth, 2009: Generation of Bulk Shear Spikes in Shallow Stratified Tidal Seas. Journal of Physical Oceanography, 39 (4), 969-985, doi:Doi10.1175/2008jpo4074.1.

Chen, S., J. A. Polton, J. Hu, and J. Xing, 2015: Local inertial oscillations in the surface ocean generated by time-varying winds. Ocean Dynamics, 65 (12), 1633-1641, doi:10.1007/ s10236-015-0899-6.

Chen, S., J. A. Polton, J. Hu, and J. Xing, 2016: Thermocline bulk shear analysis in the northern North Sea. Ocean Dynamics, 66 (4), 499-508, doi:10.1007/s10236-016-0933-3. 
D’Asaro, E. A., 1985: The Energy Flux from the Wind to Near-Inertial Motions in the Surface Mixed Layer. Journal of Physical Oceanography, 15 (8), 1043-1059, doi:10.1175/1520-0485.

D’Asaro, E. A., 2014: Turbulence in the Upper-Ocean Mixed Layer. Annual Review of Marine Science, 6 (1), 101-115, doi:10.1146/annurev-marine-010213-135138.

de Boyer Montégut, C., G. Madec, A. S. Fischer, A. Lazar, and D. Iudicone, 2004: Mixed layer depth over the global ocean: An examination of profile data and a profile-based climatology. Journal of Geophysical Research, 109 (C12003), doi:10.1029/2004JC002378.

Dohan, K., and R. E. Davis, 2011: Mixing in the Transition Layer during Two Storm Events. Journal of Physical Oceanography, 41 (1), 42-66, doi:10.1175/2010JPO4253.1.

Fairall, C. W., E. F. Bradley, D. Rogers, J. Edson, and G. Young, 1996: Bulk parameterization of air-sea fluxes for TOGA COARE. J. Geophys. Res., 101, 3747-3764.

Fer, I., A. K. Peterson, and J. E. Ullgren, 2014: Microstructure Measurements from an Underwater Glider in the Turbulent Faroe Bank Channel Overflow. Journal of Atmospheric and Oceanic Technology, 31 (5), 1128-1150, doi:10.1175/JTECH-D-13-00221.1.

Grant, A. L. M., and S. E. Belcher, 2009: Characteristics of Langmuir Turbulence in the Ocean Mixed Layer. Journal of Physical Oceanography, 39 (8), 1871-1887, doi:10.1175/ 2009jpo4119.1.

Grant, A. L. M., and S. E. Belcher, 2011: Wind-Driven Mixing below the Oceanic Mixed Layer. Journal of Physical Oceanography, 41 (8), 1556-1575, doi:10.1175/jpo-d-10-05020.1.

Haghshenas, A., and J. P. Mellado, 2019: Characterization of wind-shear effects on entrainment in a convective boundary layer. Journal of Fluid Mechanics, 858, 145-183, doi:10.1017/jfm.2018. 761. 
Inall, M. E., and A. Audsley, 2012: Cruise Report: RSS Discovery Cruise 376. Tech. rep., The Scottish Association for Marine Science, Oban, Scotland, 1-239 pp.

Johnston, T. M. S., D. Chaudhuri, M. Mathur, D. L. Rudnick, D. Sengupta, H. L. Simmons, A. Tandon, and T. O. Society, 2016: Decay Mechanisms of Near- Inertial Mixed Layer Oscillations in the Bay of Bengal. Oceanography, 29 (2), doi:http://dx.doi.org/10.5670/oceanog.2016.50.

Johnston, T. M. S., and D. L. Rudnick, 2009: Observations of the Transition Layer. Journal of Physical Oceanography, 39 (3), 780-797, doi:10.1175/2008JPO3824.1.

Kilbourne, B. F., and J. B. Girton, 2015: Quantifying High-Frequency Wind Energy Flux into Near-Inertial Motions in the Southeast Pacific. Journal of Physical Oceanography, 45 (2), 369386, doi:10.1175/JPO-D-14-0076.1.

Large, W. G., and G. B. Crawford, 1995: Observations and Simulations of Upper-Ocean Response to Wind Events during the Ocean Storms Experiment. Journal of Physical Oceanography, 25, 2831-2852, doi:10.1175/1520-0485(1995)025〈2831:OASOUO $\rangle 2.0 . C O ; 2$.

Lenn, Y.-d., T. P. Rippeth, C. P. Old, S. Bacon, V. Polyak, V. Ivanova, and J. Holemann, 2011: Intermittent Intense Turbulent Mixing under Ice in the Laptev Sea Continental Shelf. Journal of Physical Oceanography, 41 (3), 531-547, doi:Doi10.1175/2010jpo4425.1, URL http://journals. ametsoc.org/doi/abs/10.1175/2010JPO4425.1.

Li, Q., and B. Fox-Kemper, 2017: Assessing the Effects of Langmuir Turbulence on the Entrainment Buoyancy Flux in the Ocean Surface Boundary Layer. Journal of Physical Oceanography, 47, 2863-2886, doi:10.1175/JPO-D-17-0085.1. 
Lincoln, B. J., T. P. Rippeth, and J. H. Simpson, 2016: Surface mixed layer deepening through wind shear alignment in a seasonally stratified shallow sea. Journal of Geophysical Research: Oceans, doi:10.1002/2015JC011382.

Lombardo, C. P., and M. C. Gregg, 1989: Similarity scaling of viscous and thermal dissipation in a convecting surface boundary layer. Journal of Geophysical Research: Oceans, 94 (C5), 6273-6284, doi:10.1029/JC094iC05p06273.

Lueck, R. G., and J. J. Picklo, 1990: Thermal Inertia of Conductivity Cells: Observations with a Sea-Bird Cell. Journal of Atmospheric and Oceanic Technology, 7 (5), 756-768, doi:10.1175/ 1520-0426(1990)007〈0756:tiocco $\rangle 2.0 . c o ; 2$.

McWilliams, J. C., E. Huckle, J. Liang, and P. P. Sullivan, 2013: Langmuir Turbulence in Swell. Journal of Physical Oceanography, 44, 870-890, doi:10.1175/JPO-D-13-0122.1.

McWilliams, J. C., P. P. Sullivan, and C.-H. Moeng, 1997: Langmuir turbulence in the ocean. J. Fluid Mech., 334, 1-30.

Merckelbach, L., D. Smeed, and G. Griffiths, 2010: Vertical water velocities from underwater gliders. Journal of Atmospheric and Oceanic Technology, 27 (3), 547-563, doi:10.1175/ 2009JTECHO710.1.

Munk, W., 1981: Internal waves and small-scale processes. Evolution of Physical Oceanography, B. A. Warren, and C. Wunsch, Eds., Cambridge: Massachusetts Institute of Technology, 246291.

National Oceanography Centre/University of Southampton,, 2008: NOCS Surface Flux Dataset v2.0. Research Data Archive at the National Center for Atmospheric Research, Computational 
and Information Systems Laboratory, Boulder CO, URL http://rda.ucar.edu/datasets/ds260.3/ (Accessed:16July2014).

Niiler, P. P., and E. Kraus, 1977: One-dimensional models of the upper ocean. Modelling and Prediction of the Upper Layers of the Ocean, Pergamon Press, chap. Chapter 10, 143-172.

Palmer, M., G. Stephenson, M. E. Inall, C. Balfour, A. Düsterhus, and M. Green, 2015: Turbulence and Mixing by Internal Waves In The Celtic Sea Determined From Ocean Glider Microstructure Measurements. Journal of Marine Systems, 144, 57-69, doi:10.1016/j.jmarsys.2014.11.005.

Phillips, O. M., 1977: Dynamics of the Upper Ocean. Cambridge University Press, 336 pp.

Plueddemann, A. J., and J. T. Farrar, 2006: Observations and models of the energy flux from the wind to mixed-layer inertial currents. Deep-Sea Research Part II: Topical Studies in Oceanography, 53, 5-30, doi:10.1016/j.dsr2.2005.10.017.

Plueddemann, A. J., and R. A. Weller, 1999: Structure and evolution of the oceanic surface boundary layer during the Surface Waves Processes Program. Journal of Marine Systems, 21, 85-102.

Pollard, R., and R. Millard, 1970: Comparison between observed and simulated wind-generated inertial oscillations. Deep Sea Research and Oceanographic Abstracts, 17 (4), 813-821, doi: 10.1016/0011-7471(70)90043-4.

Pollard, R. T., 1980: Properties of Near-Surface Inertial Oscillations. Journal of Physical Oceanography, 10, 385-398, doi:10.1175/1520-0485(1980)010<0385:PONSIO $\rangle$ 2.0.CO;2.

Polton, J. A., D. M. Lewis, and S. E. Belcher, 2005: The Role of Wave-Induced Coriolis-Stokes Forcing on the Wind-Driven Mixed Layer. Journal of Physical Oceanography, 35, 444-457. 
Rippeth, T. P., B. J. Lincoln, H. A. Kennedy, M. Palmer, J. Sharples, and C. A. J. Williams, 2014: Impact of vertical mixing on sea surface $\mathrm{pCO} 2$ in temperate seasonally stratified shelf seas. Journal of Geophysical Research: Oceans, 119, 3863-3882, doi:10.1002/2014JC010192.

Rippeth, T. P., M. Palmer, J. H. Simpson, N. R. Fisher, and J. Sharples, 2005: Thermocline mixing in summer stratified continental shelf seas. Geophysical Research Letters, 32 (5), doi: ArtnL05602Doi10.1029/2004g1022104.

Rippeth, T. P., P. J. Wiles, M. Palmer, J. Sharples, and J. F. Tweddle, 2009: The diapcynal nutrient flux and shear-induced diapcynal mixing in the seasonally stratified western Irish Sea. Continental Shelf Research, 29 (13), 1580-1587, doi:DOI10.1016/j.csr.2009.04.009.

Rumyantseva, A., N. Lucas, T. Rippeth, A. Martin, S. C. Painter, T. J. Boyd, and S. Henson, 2015: Ocean nutrient pathways associated with the passage of a storm. Global Biogeochemical Cycles, 29, 1179-1189, doi:10.1002/2015GB005097.

Skyllingstad, E. D., W. D. Smyth, and G. B. Crawford, 2000: Resonant Wind-Driven Mixing in the Ocean Boundary Layer. Journal of Physical Oceanography, 30 (8), 1866-1890, doi: 10.1175/1520-0485(2000)030〈1866:RWDMIT $\rangle 2.0 . C O ; 2$.

Sutherland, G., K. H. Christensen, and B. Ward, 2014: Evaluating Langmuir turbulence parameterizations in the ocean surface boundary layer. Journal of Geophysical Research: Oceans, 119, 1899-1910, doi:10.1002/2013JC009537.

Thompson, A. F., A. Lazar, C. Buckingham, A. C. Naveira Garabato, G. M. Damerell, and K. J. Heywood, 2016: Open-Ocean Submesoscale Motions: A Full Seasonal Cycle of Mixed Layer Instabilities from Gliders. Journal of Physical Oceanography, 46, 1285-1307, doi:10.1175/ JPO-D-15-0170.1. 
Watanabe, M., and T. Hibiya, 2002: Global estimates of the wind-induced energy flux to inertial motions in the surface mixed layer. Geophysical Research Letters, 29 (8), 64-1, doi:10.1029/ $2001 G L 014422$.

Webb, A., and B. Fox-Kemper, 2011: Wave spectral moments and Stokes drift estimation. Ocean Modelling, 40 (3-4), 273-288, doi:10.1016/j.ocemod.2011.08.007.

Whitt, D. B., and J. R. Taylor, 2017: Energetic Submesoscales Maintain Strong Mixed Layer Stratification during an Autumn Storm. Journal of Physical Oceanography, 47, 2419-2427, doi:10.1175/JPO-D-17-0130.1.

Wu, J., 1983: Sea-Surface Drift Currents Induced by Wind and Waves. Journal of Physical Oceanography, 13 (8), 1441-1451, doi:10.1175/1520-0485(1983)013〈1441:SSDCIB $\rangle 2.0 . C O$; 2.

Zikanov, O., D. N. Slinn, and M. R. Dhanak, 2003: Large-eddy simulations of the windinduced turbulent Ekman layer. Journal of Fluid Mechanics, 495, 343-368, doi:10.1017/ S0022112003006244. 


\section{LIST OF FIGURES}

Fig. 1. Location of the Porcupine Abyssal Plain (PAP) site used for the OSMOSIS project. Panel b) shows the PAP site location, panel a) is the PAP site box-to-scale; Black crosses represent the mooring array, the green and red data shows the track of the OMG, which commenced in the North West corner and traversing roughly South East. The red colouring denotes the storm period.

Fig. 2. The environmental conditions. Panels a) and b) show the meteorological time-series: Panel a) shows the friction velocity $u_{* w}$ (black) and the Stokes drift amplitude $U_{s o}$ (blue); Panel b) shows the buoyancy flux $B_{0}$ (black) and air pressure (blue). Panels c) - d) show the oceanic time-series with the Mixed Layer Depth (MLD) and the Transition Layer Depth (TLD) displayed on each (cyan and blue respectively). Panels c) shows the profiling OMG temperature time-series with 0.5 degree Celsius contours (white). Panel d) shows the profiling OMG dissipation time-series. The vertical red dashed lines show the approximate sunrise/sunset periods and the vertical green lines show the start and end of the 'storm period'.

Fig. 3. Temperature profiles during the storm period, where the storm is split into three equal periods: early storm (black); mid-storm (blue); late-storm (red). The horizontal lines represent the mean MLD and TLD for each period. The mixed layer cools in response to the storm, while the Transition Layer warms. The MLD and TLD deepen during the storm.

Fig. 4. Comparison of velocity components from the bulk model (solid curves) and from the geographical components of the ship board ADCP. The observations are the difference between the velocity in the OSBL and the velocity at $49 \mathrm{~m}$. Note, that due to poor data return from the ship's ADCP, this data stream is intermittent. The green vertical lines again represent the storm period.

Fig. 5. Time-depth cross section of the dissipation rate during the storm obtained from the OMG. The dashed curve shows the depth of the OSBL obtained from the model, captured during the storm deepening process. The green vertical lines again represent the storm period.

Fig. 6. (a) Comparison between temperature from the bulk model (solid curve) and from the glider (crosses). The temperatures from the glider and the model curve were obtained from the average over the depth of the OSBL. The red line in figure 6(b) is estimated from a linear fit to the observed temperatures between days 268.25 and 269.75. (b) is as (a) but an expanded view of the last two days.

Fig. 7. Non-dimensional profiles of the dissipation rate from the OMG (a) between days 266.25 and 267.8 and (b) between days 268.25 and 269.8. The dissipation rate has been scaled according to the Langmuir scaling of Grant and Belcher (2009) by $w_{* L}^{3} / h_{\mathrm{ml}}$, and depth by the mixed layer depth from the temperature profiles. The blue solid curve is the dissipation rate profile from one of the LES used in Grant and Belcher (2009), the cyan solid curve is the mean of the observational OMG profiles.

Fig. 8. Time series of the dissipation rate at different depths below the base of the WML determined from the temperature profiles. The stars show the dissipation rate from the glider, where (a) $5 \mathrm{~m}$ above the base of the WML, with the curve showing the dissipation calculated using Eq. 6, (b) $5.5 \mathrm{~m}$ below the base of the WML, (c) $7.5 \mathrm{~m}$ below, (d) $9.5 \mathrm{~m}$ below. In (b) to (d) the black curves show the dissipation rates calculated from Eq. 5, multiplied by 1.0, 0.5 and 0.25 to account for the decrease in the dissipation rate with depth. The red curves after day 268.5 are just the black curves divided by two. Note the different scales on the y-axis. 
Fig. A1. Comparison of shear production from the simulation with $f=0.25 \times 10^{-4} s-1$ (crosses) and the parameterisation, Eq. A2 (curve). (b) The parameter $\alpha$ in Eq. A2 as a function of $f h_{b l} / u_{* w}$. The dotted curve is $\alpha=0.4 \exp \left(-4.5 f h_{b l} / u_{* w}\right)$. 

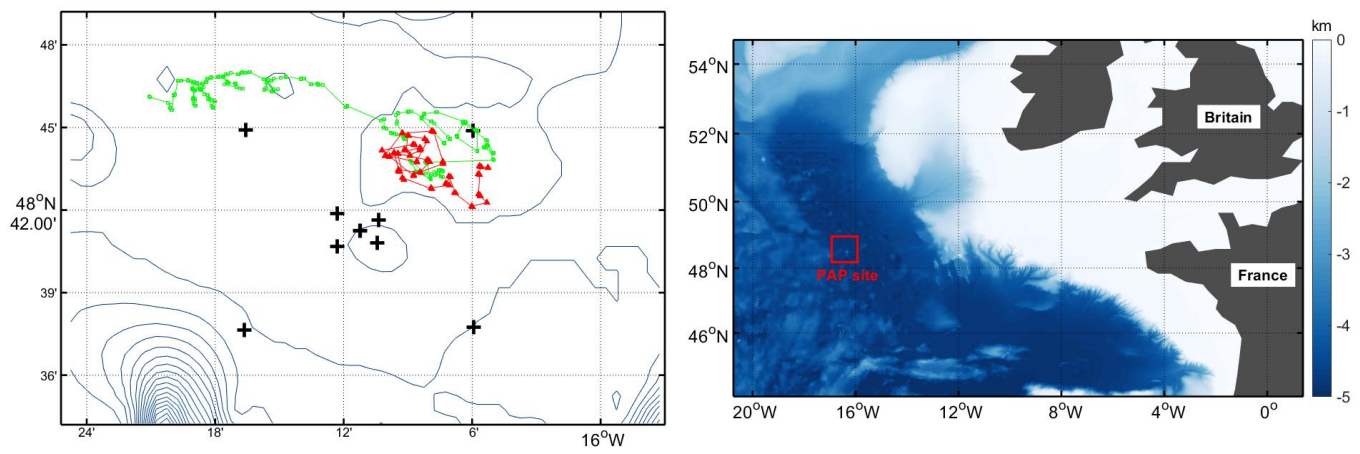

FIG. 1. Location of the Porcupine Abyssal Plain (PAP) site used for the OSMOSIS project. Panel b) shows the PAP site location, panel a) is the PAP site box-to-scale; Black crosses represent the mooring array, the green and red data shows the track of the OMG, which commenced in the North West corner and traversing roughly 

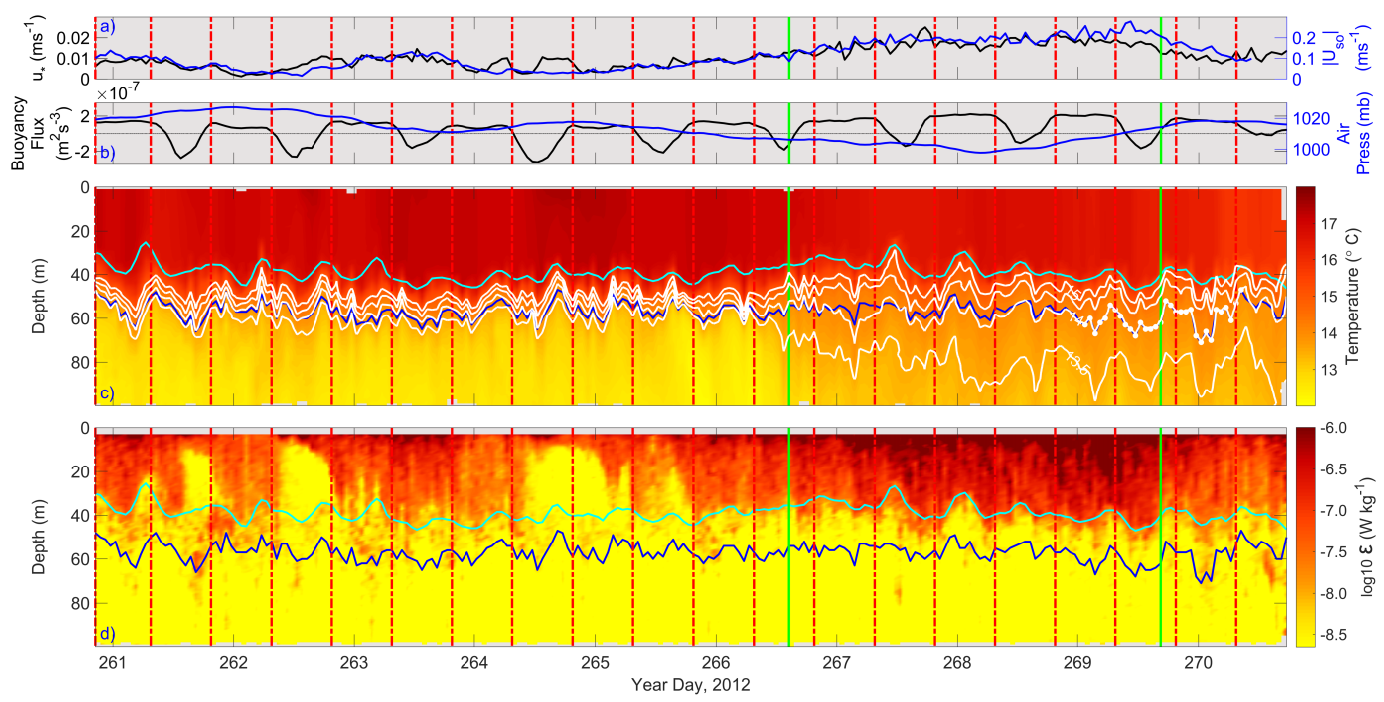

FIG. 2. The environmental conditions. Panels a) and b) show the meteorological time-series: Panel a) shows the friction velocity $u_{* w}$ (black) and the Stokes drift amplitude $U_{s o}$ (blue); Panel b) shows the buoyancy flux $B_{0}$ (black) and air pressure (blue). Panels c) - d) show the oceanic time-series with the Mixed Layer Depth (MLD) and the Transition Layer Depth (TLD) displayed on each (cyan and blue respectively). Panels c) shows the profiling OMG temperature time-series with 0.5 degree Celsius contours (white). Panel d) shows the profiling OMG dissipation time-series. The vertical red dashed lines show the approximate sunrise/sunset periods and the vertical green lines show the start and end of the 'storm period'. 


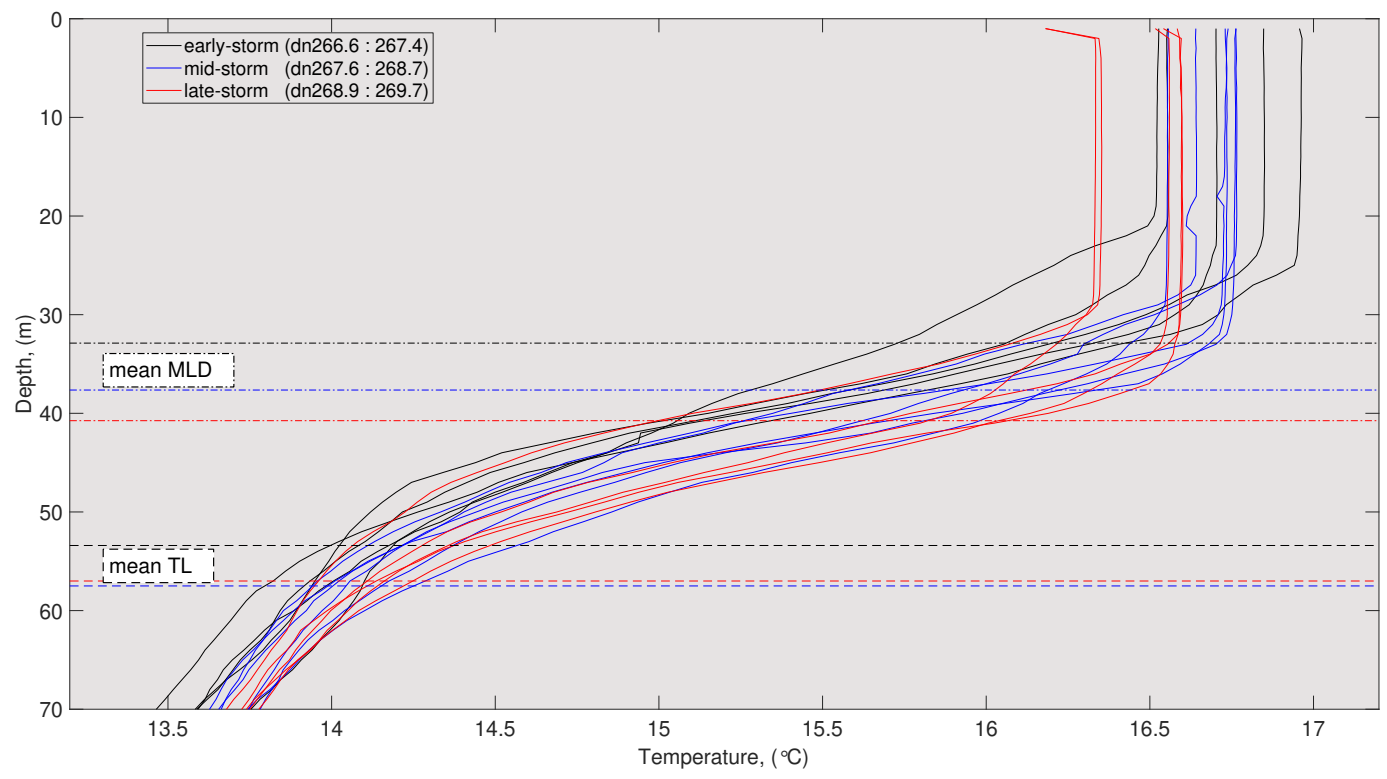

FIG. 3. Temperature profiles during the storm period, where the storm is split into three equal periods: early storm (black); mid-storm (blue); late-storm (red). The horizontal lines represent the mean MLD and TLD for each period. The mixed layer cools in response to the storm, while the Transition Layer warms. The MLD and TLD deepen during the storm. 

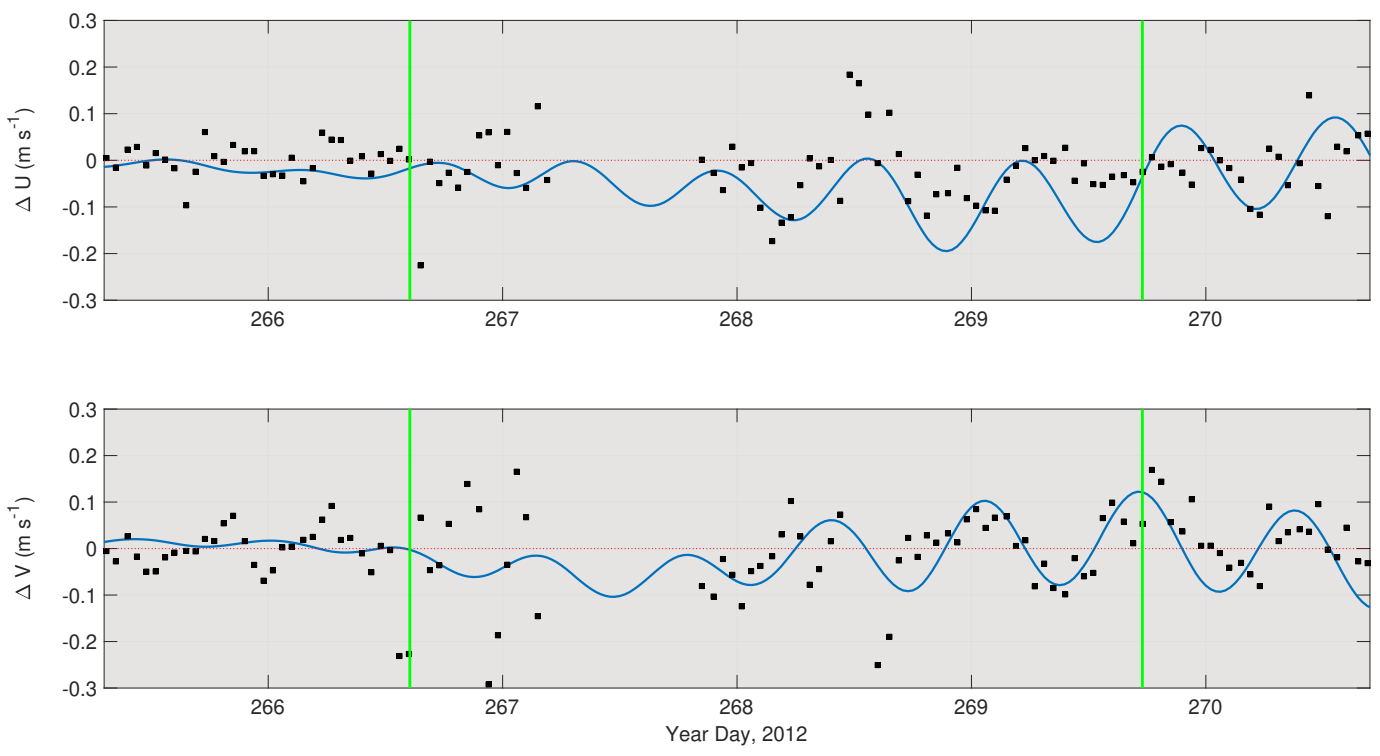

FIG. 4. Comparison of velocity components from the bulk model (solid curves) and from the geographical components of the ship board ADCP. The observations are the difference between the velocity in the OSBL and the velocity at $49 \mathrm{~m}$. Note, that due to poor data return from the ship's ADCP, this data stream is intermittent. 


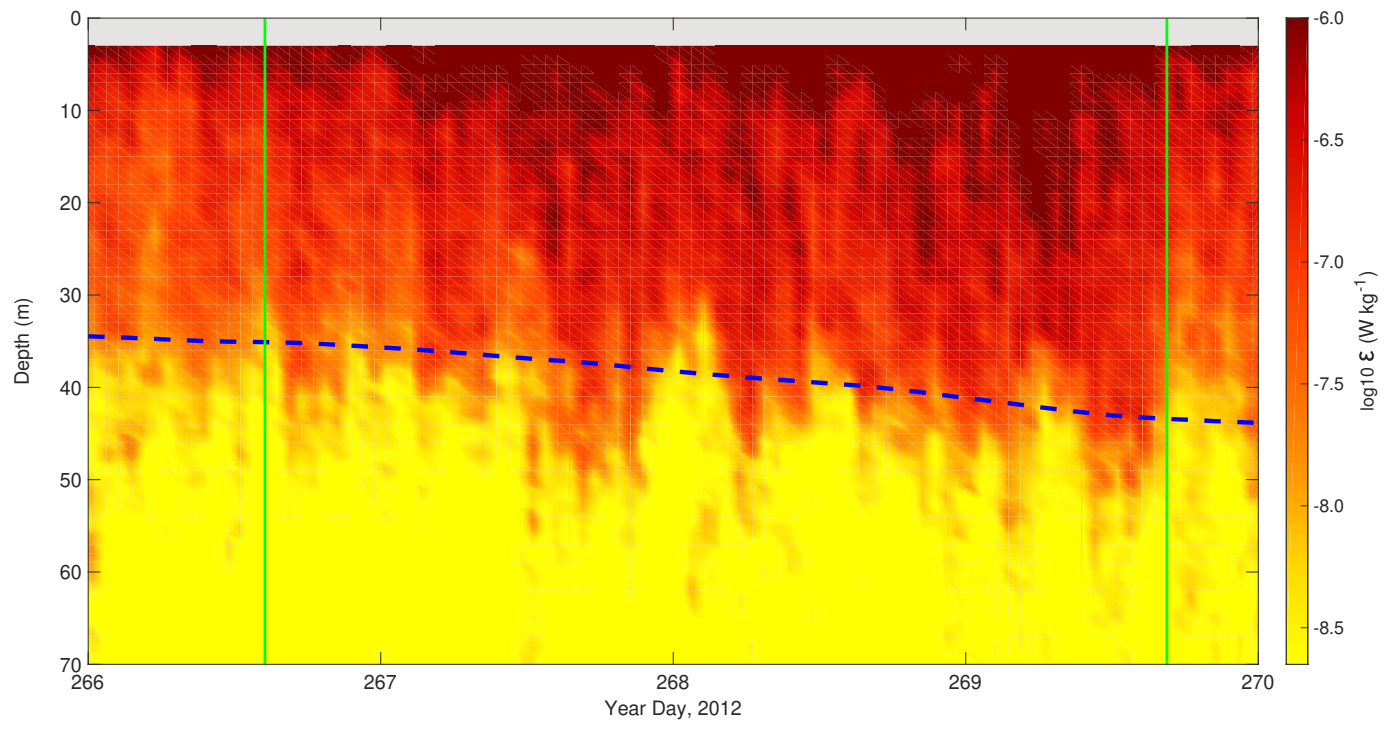

FIG. 5. Time-depth cross section of the dissipation rate during the storm obtained from the OMG. The dashed curve shows the depth of the OSBL obtained from the model, captured during the storm deepening process. The green vertical lines again represent the storm period. 

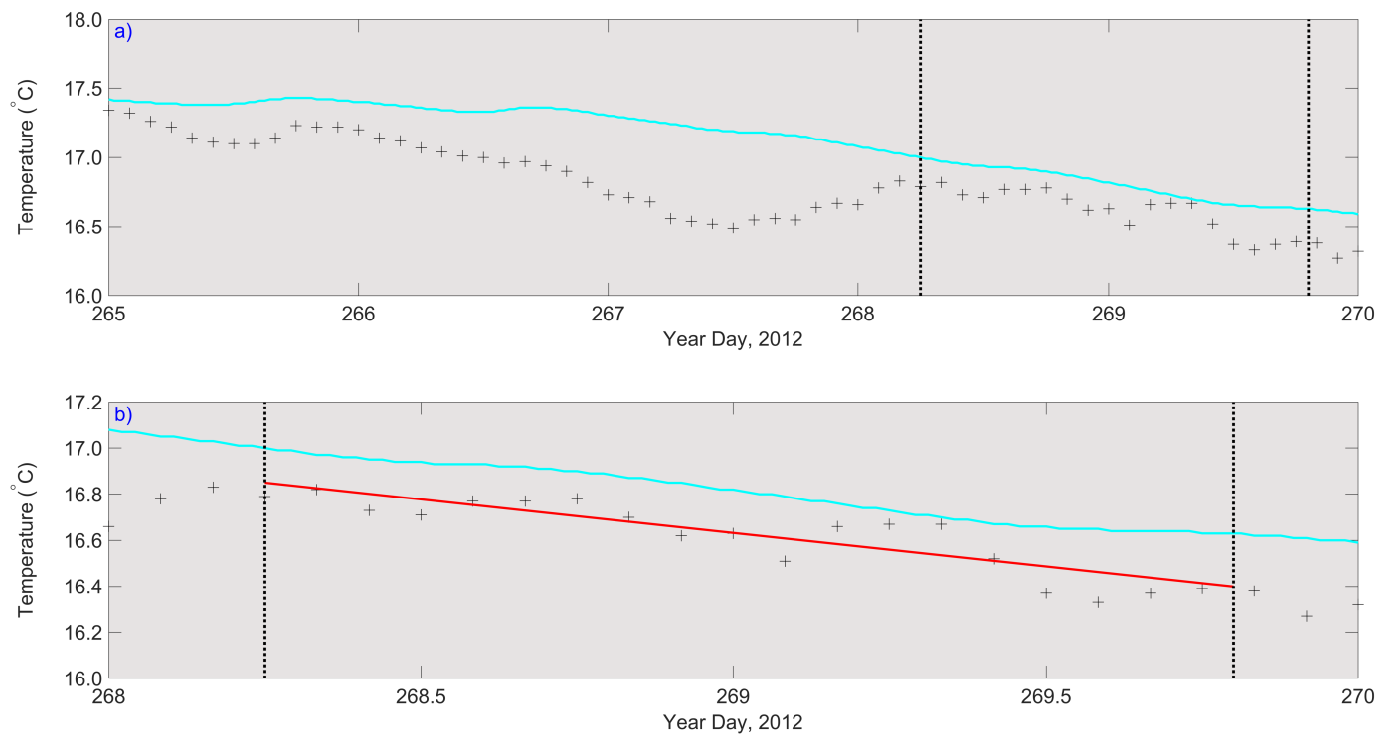

FIG. 6. (a) Comparison between temperature from the bulk model (solid curve) and from the glider (crosses). The temperatures from the glider and the model curve were obtained from the average over the depth of the OSBL. The red line in figure 6(b) is estimated from a linear fit to the observed temperatures between days 268.25 and 269.75. (b) is as (a) but an expanded view of the last two days. 

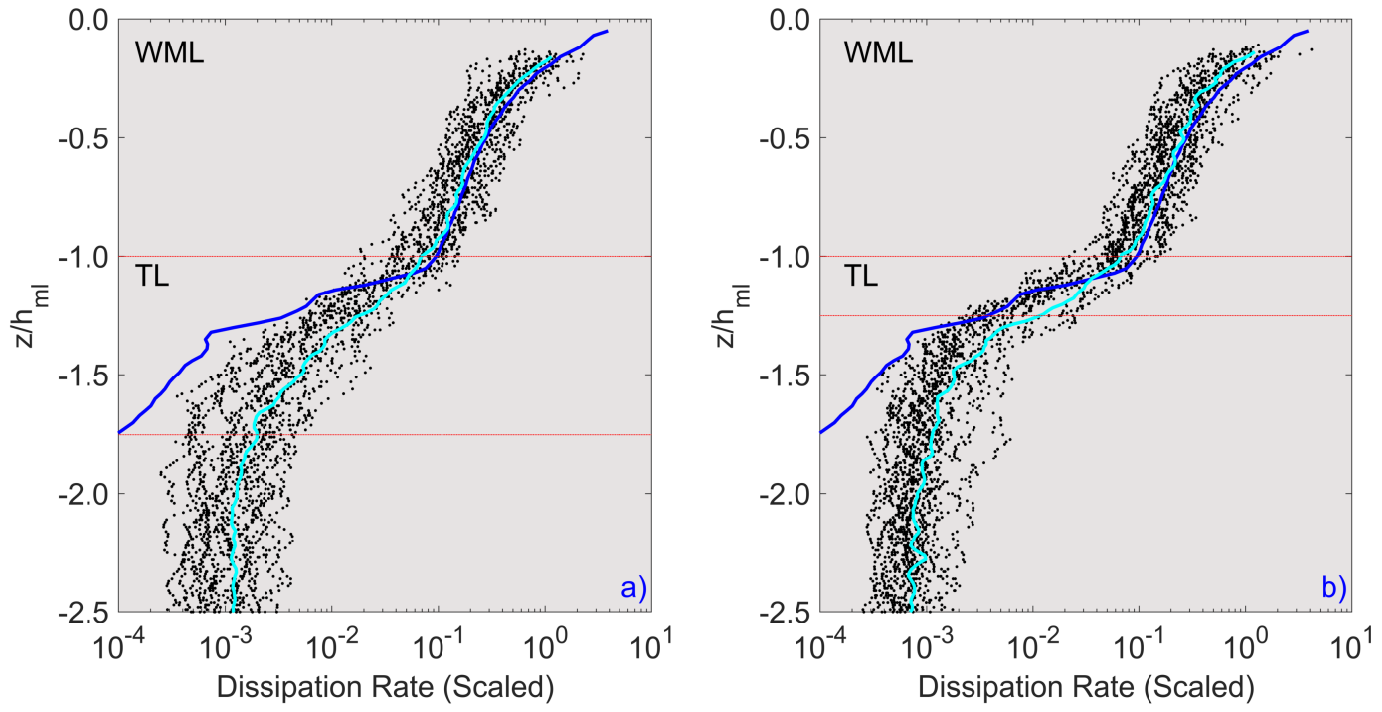

FIG. 7. Non-dimensional profiles of the dissipation rate from the OMG (a) between days 266.25 and 267.8 and (b) between days 268.25 and 269.8. The dissipation rate has been scaled according to the Langmuir scaling of Grant and Belcher (2009) by $w_{* L}^{3} / h_{\mathrm{ml}}$, and depth by the mixed layer depth from the temperature profiles. The blue solid curve is the dissipation rate profile from one of the LES used in Grant and Belcher (2009), the cyan solid curve is the mean of the observational OMG profiles. 

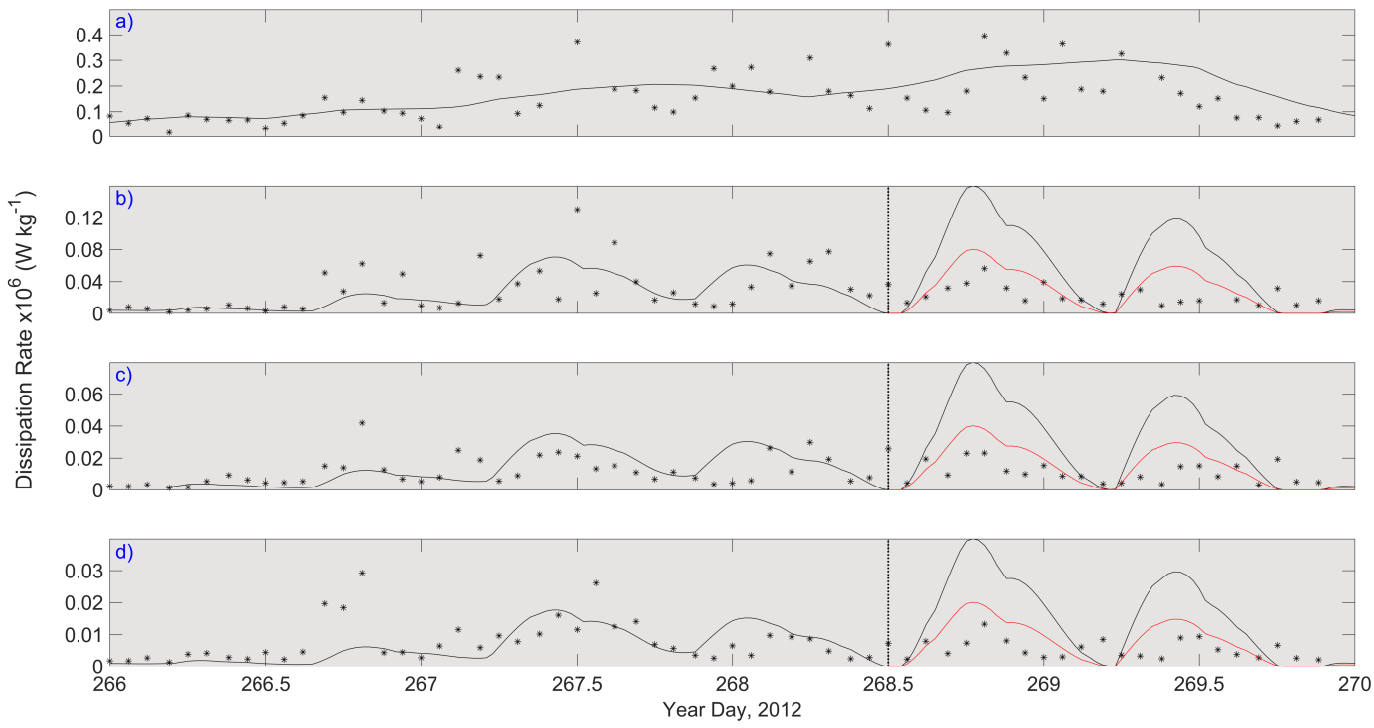

FIG. 8. Time series of the dissipation rate at different depths below the base of the WML determined from the temperature profiles. The stars show the dissipation rate from the glider, where (a) $5 \mathrm{~m}$ above the base of the WML, with the curve showing the dissipation calculated using Eq. 6, (b) 5.5m below the base of the WML, (c) $7.5 \mathrm{~m}$ below, (d) $9.5 \mathrm{~m}$ below. In (b) to (d) the black curves show the dissipation rates calculated from Eq. 5 , multiplied by $1.0,0.5$ and 0.25 to account for the decrease in the dissipation rate with depth. The red curves after day 268.5 are just the black curves divided by two. Note the different scales on the y-axis. 

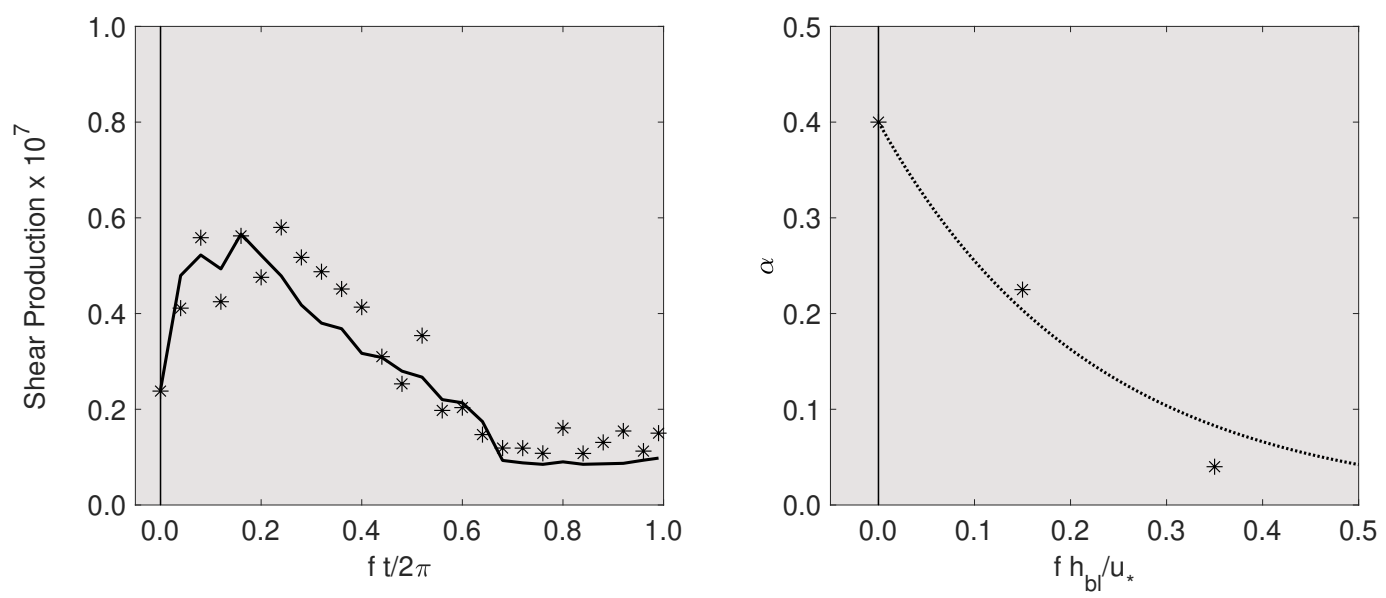

Fig. A1. Comparison of shear production from the simulation with $f=0.25 \times 10^{-4} s-1$ (crosses) and the parameterisation, Eq. A2 (curve). (b) The parameter $\alpha$ in Eq. A2 as a function of $f h_{b l} / u_{* w}$. The dotted curve is $\alpha=0.4 \exp \left(-4.5 f h_{b l} / u_{* w}\right)$. 